\title{
A novel allele of TaGW2-A1 is located in a finely mapped QTL that increases grain weight but decreases grain number in wheat (Triticum aestivum $\mathrm{L}$. )
}

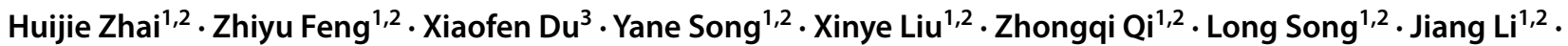

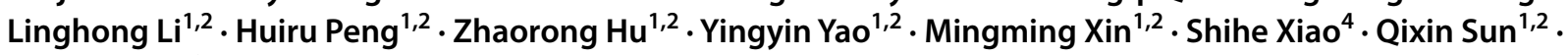 \\ Zhongfu $\mathrm{Ni}^{1,2}$
}

Received: 16 May 2017 / Accepted: 4 November 2017 / Published online: 17 November 2017

(c) The Author(s) 2017. This article is an open access publication

\begin{abstract}
Key message A novel TaGW2-A1 allele was identified from a stable, robust QTL region, which is pleiotropic for thousand grain weight, grain number per spike, and grain morphometric parameters in wheat.

Abstract Thousand grain weight (TGW) and grain number per spike (GNS) are two crucial determinants of wheat spike yield, and genetic dissection of their relationships can help to fine-tune these two components and maximize grain yield. By evaluating 191 recombinant inbred lines in 11 field trials, we identified five genomic regions on chromosomes 1B, $3 \mathrm{~A}, 3 \mathrm{~B}, 5 \mathrm{~B}$, or 7A that solely influenced either TGW or GNS, and a further region on chromosome $6 \mathrm{~A}$ that concurrently affected TGW and GNS. The QTL of interest on chromosome 6A, which was flanked by wsnp_BE490604A_Ta_2_1 and wsnp_RFL_Contig1340_448996 and designated as $Q T g w / G n s . c a u-6 A$, was finely mapped to a genetic interval shorter than $0.538 \mathrm{cM}$ using near isogenic lines (NILs). The elite NILs of $Q T g w / G n s . c a u-6 A$ increased TGW by $8.33 \%$, but decreased GNS by $3.05 \%$ in six field trials. Grain Weight 2 (TaGW2-A1), a well-characterized gene that negatively regulates TGW and grain width in wheat, was located within the finely mapped interval of $Q T g w / G n s . c a u-6 A$. A novel and rare $T a G W 2-A 1$ allele with a 114-bp deletion in the 5' flanking region was identified in the parent with higher TGW, and it reduced TaGW2-Al promoter activity and expression. In conclusion, these results expand our knowledge of the genetic and molecular basis of TGW-GNS trade-offs in wheat. The QTLs and the novel $T a G W 2-A l$ allele are likely useful for the development of cultivars with higher TGW and/or higher GNS.
\end{abstract}

Communicated by Diane E. Mather.

Electronic supplementary material The online version of this article (https://doi.org/10.1007/s00122-017-3017-y) contains supplementary material, which is available to authorized users.

Zhongfu Ni

nizf@cau.edu.cn

1 State Key Laboratory for Agrobiotechnology, Key Laboratory of Crop Heterosis and Utilization, Beijing Key Laboratory of Crop Genetic Improvement, China Agricultural University, Beijing 100193, China

2 National Plant Gene Research Centre, Beijing 100193, China

3 Millet Research Institute, Shanxi Academy of Agricultural Sciences, Changzhi 046011, Shanxi, China

4 Institute of Crop Science, Chinese Academy of Agricultural Sciences, Beijing 100081, China

\section{Introduction}

Wheat provides approximately $20 \%$ of the calories consumed by humankind (Simmonds et al. 2016). Considering the continued global population growth and the low rates of genetic gain in wheat yield (Zheng et al. 2011; Ray et al. 2013), there is an urgent need to identify, characterize, and incorporate genomic tools that can accelerate wheat yield improvement (Simmonds et al. 2016). Wheat yield is controlled by polygenes and affected by environmental factors, and it mainly relies on three components: thousand grain weight (TGW), grain number per spike (GNS), and spike number per hectare (Simmonds et al. 2014). There are negative correlations among these components (Griffiths et al. 2015), but the genetic basis underlying individual yield traits and their interactions are still largely unknown in wheat.

In rice, it has been shown that grain weight is affected by the genes that functioning in several pathways, i.e., proteasomal 
degradation (GW2 and GW5/qSW5), phytohormone signaling (GS6, TGW6, and OsCKX2), and G protein-mediated signal transduction (GS3 and $R G B 1$ ); these genes regulate cell division and/or cell expansion in specific grain tissues (reviewed by Zuo and Li 2014). Using a homology-based approach, several wheat genes have been isolated and suggested to be associated with TGW. These include TaCwi (Jiang et al. 2015b; Ma et al. 2012), TaTGW6 (Hanif et al. 2016; Hu et al. 2016), TaGW2-Al (Simmonds et al. 2016; Su et al. 2011; Yang et al. 2012), TaGSIa (Guo et al. 2013), TaGS5-3A (Ma et al. 2016), TaGASR7-Al (Dong et al. 2014; Ling et al. 2013) and TaCYP78A3 (Ma et al. 2015). Research on these genes has enhanced our understanding on grain weight determination in wheat and has also provided functional markers useful for selecting higher TGW through marker-assisted breeding.

Several major QTLs for grain number have also been isolated and characterized in rice. Some of these QTLs control inflorescence meristem identity (e.g., $L A X I, S P A$, and $F Z P$ ), while others influence the rate (e.g., APO1, Gnla/OsCKX2, LOG, $S P 1$, and $D E P 1$ ) or duration (e.g., RCN1, RCN2, Ghd7, and $G h d 8$ ) of cell division in the inflorescence meristem (reviewed by Xing and Zhang 2010). Although the functions of orthologous genes are generally conserved in rice and wheat, there are several examples of functional divergence between rice grain number genes and their wheat homologs. For instance, OsCKX2, encoding a cytokinin oxidase, is a negative regulator of the number of grains per panicle in rice (Ashikari et al. 2005). On the other hand, the two wheat homologs of $O_{s} C K X 2$, i.e., $T a C K X 2.1$ and $T a C K X 2.2$, have been suggested to positively control GNS (Zhang et al. 2011). Furthermore, while MOC1 and $O S T E F 1$ are two crucial regulators of rice tillering (Li et al. 2003; Paul et al. 2012), their wheat homologs (TaMOC1-7A and TaTEF-7A) have both been found to be stably associated with spikelet number per spike rather than with tiller number (Zhang et al. 2015; Zheng et al. 2014). Differences such as these may be associated with the contrasting architecture of the inflorescences between wheat (spikes) and rice (panicles).

Here, we report mapping of several stable QTLs for TGW and/or GNS using 191 recombinant inbred lines (RILs) derived from a cross between two wheat lines that differ in both TGW and GNS. Of these QTLs, the one on chromosome 6A displayed a strong TGW-GNS trade-off. This QTL was further validated and precisely mapped using near isogenic lines (NILs). A novel allele of TaGW2-Al was isolated from the finely mapped interval, which is likely a candidate.

\section{Materials and methods}

\section{Plant materials}

A population of 207 RILs was developed from the cross between two Chinese hexaploid winter wheat cultivars, i.e.,
Yumai 8679 (Y8679) and Jing 411 (J411), and advanced to the $\mathrm{F}_{9}$ generation by single seed descent. Of the 207 RILs, 191 were genotyped and evaluated in 11 field trials. An $\mathrm{F}_{9}$ plant of the RIL line (RIL186) with residual heterozygosity at the marker locus Xbarc118 was selfed and provided an $\mathrm{F}_{9: 10}$ family with 163 progeny. Afterwards, selfing of these plants was carried out to obtain a population of $163 \mathrm{~F}_{10: 11}$ families. Four sets of NIL pairs (NIL1, NIL2, NIL3, and NIL4) were obtained by selfing selected $F_{11}$ progeny with overlapping heterozygous fragments. Each NIL set comprised eight to 25 Y8679-type homozygotes and 12-30 J411-type homozygotes.

In total, 1113 wheat accessions with varying ploidy were used to test the allele frequency of a novel TaGW2-Al allele. These included 848 hexaploid wheat accessions (96 Chinese landraces, 702 Chinese modern cultivars, and 50 French varieties; Supplementary Table S1), 238 tetraploid accessions (181 Triticum dicoccoides, 33 Triticum durum, and 24 Triticum dicoccum; Supplementary Table S2), and 27 diploid accessions (6 Triticum urartu, 6 Triticum boeoticum, and 15 Triticum monococcum; Supplementary Table S3).

\section{Field experiments and phenotyping}

The RIL population was grown in three replicates following randomized complete-block design at the following five experimental sites: Anhui $\left(33^{\circ} 48^{\prime} \mathrm{N}, 116^{\circ} 35^{\prime} \mathrm{E}\right)$, Beijing $\left(40^{\circ} 08^{\prime} \mathrm{N}, 116^{\circ} 10^{\prime} \mathrm{E}\right)$, Hebei $\left(37^{\circ} 56^{\prime} \mathrm{N}, 114^{\circ} 42^{\prime} \mathrm{E}\right)$, Shaanxi $\left(34^{\circ} 17^{\prime} \mathrm{N}, 108^{\circ} 04^{\prime} \mathrm{E}\right)$, and Shanxi $\left(36^{\circ} 08^{\prime} \mathrm{N}, 111^{\circ} 34^{\prime} \mathrm{E}\right)$ (Supplementary Fig. S1; Supplementary Table S4). The field trials were carried out during two crop seasons (2011-12 and 2012-13) at Anhui, four crop seasons (2010-11, 2011-12, 2012-13, and 2014-15) at Beijing, three crop seasons (2011-12, 2012-13 and 2013-14) at Shaanxi, and one crop season (2013-14 or 2014-15) at the other two sites. For the field trials, the seeds were sown in double-row plots $(2 \mathrm{~m}$ long) at a sowing rate of 30 seeds per row. At maturity, 20 representative spikes (from 20 different plants) were sampled from each plot and threshed together. For the grain samples obtained from the first two crop seasons (2010-11 and 2011-12), GNS, TGW, and grain weight per spike (GWS) were manually assessed. For the samples obtained from the other three seasons (2012-13, 2013-14, and 2014-15), seven traits [i.e., GNS, TGW, GWS, grain surface area (GA), grain circumference (GC), grain length (GL), and grain width $(\mathrm{GW})]$ were recorded using a scaled cameraassisted phenotyping system (Wanshen Detection Technology Co., Ltd., Hangzhou, China). For each RIL line, the mean value of each trait was calculated across three replicates for each individual environment. In addition, best linear unbiased prediction (BLUP) values were predicted for each trait using the PROC MIXED procedure in SAS v9.1.3 (SAS Institute Inc., North Carolina, USA). Pearson's 
correlation coefficient was calculated using SPSS v20.0 (SPSS, Chicago, USA) to assess the correlation among TGW, GNS, GWS, and grain morphometric parameters. The Shapiro-Wilk test was performed using R v3.2.2 to identify departures from normal distribution. Broad sense heritability $\left(h_{\mathrm{B}}^{2}\right)$ based on a family mean basis was calculated using the PROC GLM procedure in SAS according to the following formula: $h_{\mathrm{B}}^{2}=\sigma_{\mathrm{g}}^{2} /\left(\sigma_{\mathrm{g}}^{2}+\sigma_{\mathrm{ge}}^{2} / n+\sigma^{2} / n r\right)$, where $\sigma_{\mathrm{g}}^{2}$ is the genotypic effect, $\sigma_{\mathrm{ge}}^{2}$ is the genotype by environmental effect, $\sigma^{2}$ is the residual error, $n$ is the number of environments and $r$ is the number of replicates (Liu et al. 2014).

The NIL pairs were evaluated at three sites (Beijing, Hebei, and Shanxi) in crop seasons 2014-15 (NIL1) and 2015-16 (NIL1, NIL2, NIL3, and NIL4). The $163 \mathrm{~F}_{10: 11}$ families were evaluated at Beijing during crop season 2014-15. These NIL pairs and families were grown in single-row plots ( $2 \mathrm{~m}$ long and 30 seeds per row) in randomized complete-block designs with three replicates. At maturity, 20 representative spikes were sampled, and the GWS, GNS, TGW, and grain morphometric parameters (GA, GC, GL, and $\mathrm{GW}$ ) were assessed. For the members of NIL1 pair, tiller number (TN) and grain weight per plant (GWP) were determined for each of ten representative plants. The significance of phenotypic variations between NIL1-Y8679 and NIL1-J411 was calculated using Student's $t$ test.

\section{Linkage map and QTL analysis}

The Y8679/J411 linkage map used here is the one that was described by Zhai et al. (2016). It includes both single-nucleotide polymorphism (SNP) markers and simple sequence repeat (SSR) markers. QTL analysis was conducted using both the within-environment means and the across-environment BLUPs for each trait. The methods used for QTL analysis were the same as those used by Zhai et al. (2016) for other traits in the same population. Briefly, this involved the use of WinQTLCart version 2.5 (Wang et al. 2012) for composite interval mapping (CIM) using model 6 with forward and backward regression, five markers as cofactors, and a 10-cM scanning window. Empirical threshold LOD scores estimated with 1000 permutations at $P \leq 0.05$ were used to declare a significant QTL (Churchill and Doerge 1994). Detected QTLs with overlapping confidence intervals ( \pm 2 LOD away from the peaks of likelihood ratios) were considered equivalent and named as suggested by McIntosh et al. (2011).

For a QTL of interest on chromosome 6A, the 163 $F_{10: 11}$ families derived from RIL186 were assigned to three genotypic classes (two homozygous families and one segregating families) based on their marker genotypes. This classification was used to estimate the additive effect $(a)$, dominance effect $(d)$, and dominance degree $(d / a)$ (Falconer and Mackay 1996). Multiple comparisons among the mean values were estimated by the LSD method. The inheritance mode of an individual QTL can be classified into four categories, i.e., additive $(d / a \leq 0.20)$, partial dominance $(0.20<d / a<0.80)$, dominance $(0.80 \leq d / a<1.20)$, and overdominance $(d / a \geq 1.20)$, as described in the previous studies (Jiang et al. 2015a; Li et al. 2017).

The SNPs mapped in the QTL regions of interest were positioned onto the newly released reference genome sequence of Chinese Spring by blasting their flanking sequences against the IWGSC RefSeq v1.0 (https://urgi. versailles.inra.fr/blast_iwgsc/blast.php).

\section{SSR marker development}

Polymorphic SNP markers flanking the QTL region of interest were used to perform a BLAST search against genomic sequences of Brachypodium, rice, and Aegilops tauschii, to identify orthologous genomic regions (Supplementary Table S5) using the methods described by Zhai et al. (2016). The genes within the corresponding regions were used to search the hexaploid wheat cv. Chinese Spring IWGSC survey sequences (http://www.wheatgenome.org) to find homologous contig sequences for marker development (Lu et al. 2016). These contig sequences were further used to search for SSR motifs (with at least 15 dinucleotide or trinucleotide repeats) and design PCR primers using BatchPrimer3 (http://probes.pw.usda.gov/batchprimer3). For polymorphism detection, PCR products were separated on $8 \%$ non-denaturing PAGE gels and visualized with silver staining. The primer pairs of 14 co-dominant SSR markers are listed in Supplementary Table S6.

\section{RNA extraction, CDNA synthesis, and qRT-PCR}

For cDNA cloning analysis, the seedling leaves of Y8679 and J411 were collected for total RNA extraction at 7 days after germination (dag). For expression analysis, the immature grains of NIL1-Y8679 and NIL1-J411 were collected for total RNA extraction at 11 days after pollination (dap) using the TransZol Plant Kit (TransGen Biotech). Firststrand cDNA was synthesized using M-MLV Reverse Transcriptase (Promega, WI, USA) according to the manufacturer's instructions.

qRT-PCR was performed to quantify the $T a G W 2-A 1$ transcripts in three independent biological repeats using the homoeolog-specific primer pair TaGW2A-Q (Hong et al. 2014) (Supplementary Table S6). qRT-PCR cycling was conducted using SYBR Green PCR master mix (TaKaRa, Japan) on a BioRad CFX96 system (CA, USA) using the following thermal profile: $95^{\circ} \mathrm{C}$ for $7 \mathrm{~min}, 40$ cycles at $95^{\circ} \mathrm{C}$ for $10 \mathrm{~s}, 56{ }^{\circ} \mathrm{C}$ for $20 \mathrm{~s}$, and $72{ }^{\circ} \mathrm{C}$ for $20 \mathrm{~s}$. For each biological sample, three technical replicates were performed 
for TaGW2-Al and Actin (Supplementary Table S6). After normalizing to the endogenous control, the transcript levels were determined using the $2^{-\Delta C_{t}}$ method.

\section{Isolation of full-length TaGW2-A1 cDNA}

The complete coding sequences (CDS) of $T a G W 2$ genes were obtained from the cDNA of Y8679 and J411 using the primer pair TaGW2-1 (Su et al. 2011) (Supplementary Table S6). PCR products were gel-purified and cloned into the pGEM-T Easy Vector (Promega). The identities of three homoeologous coding sequences were determined by blasting against the wheat database (http://plants.ensembl.org/ common/Tools/Blast?db=core; Bolser et al. 2015) (Supplementary Fig. S2). The upstream and downstream regions of TaGW2-A1 in J411 were obtained using a rapid amplification of the cDNA ends (RACE) with the GeneRacer Kit (Invitrogen). The gene specific primers for 5'-RACE and 3'-RACE are listed in Supplementary Table S6.

\section{Promoter isolation and activity analysis of TaGW2-A1}

Three A-genome-specific primer pairs, including Hap-6A-P1 (Su et al. 2011), TaGW2-A1_InDel, and TaGW2-A1_ProF3 (Supplementary Table S6), were used to amplify a region of approximately $2.0-\mathrm{kb}$ upstream from the initiation codon (ATG) in the TaGW2-Al gene by PCR. The promoters of 1927 and 2041 bp length were obtained from Y8679 and J411, respectively, with a 114-bp deletion existing in the promoter obtained from Y8679.

To assess the effects of this 114-bp deletion on the promoter activity, promoter fragments from Y8679 (1121 bp) and J411 (1235 bp) were amplified (Supplementary Fig. S3) and fused with the $\beta$-glucuronidase (GUS) reporter gene sequence in the pBGWFS7.0 vector using the Gateway ${ }^{\circledR}$ system following the manufacturer's instructions (Invitrogen, CA, USA). The plasmids were transferred into the Agrobacterium tumefaciens strain GV3101. The leaves of 7-week-old tobacco (Nicotiana benthamiana) plants were infiltrated with the Agrobacterium clones. After 3 days, the total protein was extracted from 1.0-cm leaf discs (without the central veins), and the specific GUS activity of these extracts was determined using a colorimetric assay according to Leborgne-Castel et al. (1999). Briefly, the protein extracts were incubated with $p$-nitrophenyl $\beta$-D-glucuronide (PNPG) substrate at $37{ }^{\circ} \mathrm{C}$ and absorbance of the $p$-nitrophenol (PNP) product was measured at $410 \mathrm{~nm}$ (Pawar et al. 2017). Leaves infiltrated with the empty vector were used as controls. Total protein content in the extracts was quantified using the Bradford protein assay.

\section{Results}

\section{Phenotypic performance of the RIL population}

The population means and ranges of the seven investigated traits are listed in Table 1. Compared with J411, Y8679 had higher TGW and GWS, larger grain size (GA, GC, GL, and GW), and lower GNS (Supplementary Fig. S4). TGW displayed an obvious shift towards higher values in the Y8679/ J411 RIL population, whereas the other six traits exhibited normal distributions (Supplementary Fig. S5). All traits had broad sense heritabilities over 0.80 . Pearson's correlation coefficients among the seven traits were calculated based on the BLUP values across seven shared environments (i.e., E5, E6, E7, E8, E9, E10, and E11), which showed that GNS was strongly and negatively correlated with TGW, GA, GC, GL, and GW (Supplementary Table S7).
Table 1 Parental and population means, ranges, and broad sense heritabilities for TGW, GNS, GWS, and four grain morphometric parameters

\begin{tabular}{|c|c|c|c|c|c|c|c|}
\hline \multirow[t]{2}{*}{ Trait } & \multicolumn{3}{|c|}{ Parental lines } & \multicolumn{4}{|c|}{ RIL population } \\
\hline & J411 & Y8679 & Delta $(\%)$ & Min & Max & Mean \pm SD & $h_{\mathrm{B}}^{2}$ \\
\hline TGW & 47.56 & 64.95 & 36.54 & 42.36 & 65.23 & $55.73 \pm 4.21$ & 0.95 \\
\hline GNS & 50.22 & 43.26 & -13.87 & 36.42 & 51.70 & $43.84 \pm 2.95$ & 0.90 \\
\hline GWS & 2.41 & 2.74 & 13.69 & 2.00 & 2.89 & $2.47 \pm 0.17$ & 0.87 \\
\hline GA & 17.49 & 22.80 & 30.39 & 16.68 & 22.53 & $19.82 \pm 1.09$ & 0.93 \\
\hline GC & 16.89 & 20.05 & 18.67 & 16.95 & 19.89 & $18.41 \pm 0.59$ & 0.96 \\
\hline GL & 6.54 & 7.95 & 21.66 & 6.53 & 7.88 & $7.22 \pm 0.27$ & 0.97 \\
\hline GW & 3.37 & 3.62 & 7.58 & 3.14 & 3.71 & $3.47 \pm 0.11$ & 0.90 \\
\hline
\end{tabular}

The traits include thousand grain weight (TGW), grain number per spike (GNS), grain weight per spike (GWS), grain surface area (GA), grain circumference (GC), grain length (GL), and grain width (GW). Delta refers to the difference between Y8679 and J411 phenotypes as a percentage of J411. Broad sense heritability based on a family mean basis was estimated across all evaluated environments for each trait. TGW and GNS were evaluated in 11 environments (from E1 to E11), whereas the four grain morphometric parameters were evaluated in seven environments (from E5 to E11) 


\section{Identification of genomic regions harboring stable QTLs for TGW, GNS, GA, GC, GL, and GW}

QTLs repeatedly detected in $\geq 3$ individual environments and in the analysis of BLUPs were considered to be stable. According to this criterion, 34 stable QTLs for TGW, GNS, GA, GC, GL, and GW were mapped within eight genomic regions (Table 2; Supplementary Table S8). The corresponding physical intervals of these genomic regions in the Chinese Spring RefSeq v1.0 sequence are listed in Table 2 and Supplementary Table S9. Further 123 putative QTLs for TGW, GNS, GA, GC, GL, and GW that did not meet this criterion are listed in Supplementary Table S10. Of the 29 QTLs for GWS, two were detected in three (QGws.cau-1B.3) or four (QGws.cau-4D.1) environments, but not in the analysis of BLUPs (Supplementary Table S10).

The stable QTL regions on chromosomes 1B, 3A, and 7A were found to have an effect on GNS, but with no significant effect on TGW (Fig. 1; Table 2). The region on chromosome

Table 2 Eight genomic regions harboring stable QTLs for TGW, GNS, GA, GC, GL, and GW in the Y8679/J411 population

\begin{tabular}{|c|c|c|c|c|c|c|}
\hline $\begin{array}{l}\text { Chromo- } \\
\text { some }\end{array}$ & Interval $(\mathrm{cM})^{\mathrm{a}}$ & Interval $(\mathrm{Mb})^{\mathrm{b}}$ & Associated trait ${ }^{\mathrm{c}}$ & Included QTL ${ }^{\mathrm{d}}$ & Detected environment $^{\mathrm{e}}$ & References \\
\hline \multirow[t]{3}{*}{ 1B } & $0.0-7.7$ & & GNS (Y) & QGns.cau-1B.1 & E3/E7/E10/E11/C & $\begin{array}{l}\text { Griffiths et al. } \\
\text { (2015) }\end{array}$ \\
\hline & & & GWS (Y) & QGws.cau-1B.3 & & \\
\hline & & & GW (Y) & $Q G w . c a u-1 B .1$ & E6/E9/E11/C & \\
\hline \multirow[t]{3}{*}{$2 \mathrm{~B}$} & 117.0-129.6 & $691.78-727.21$ & GA (Y) & QGa.cau-2B.1; QGa.cau-2B.2 & E6/E7/E8/C & \\
\hline & & & GC (Y) & QGc.cau-2B.1; QGc.cau-2B.2 & E5/E7/E8/E9/E11/C & \\
\hline & & & GL (Y) & QGl.cau-2B.2; QGl.cau-2B.3 & $\begin{array}{l}\text { E5/E6/E7/E8/E9/E10/ } \\
\text { E11/C }\end{array}$ & Sun et al. (2009) \\
\hline $3 \mathrm{~A}$ & $30.7-34.9$ & $639.09-650.43$ & GNS (J) & QGns.cau-3A.1 & $\mathrm{E} 1 / \mathrm{E} 7 / \mathrm{E} 11 / \mathrm{C}$ & Cui et al. (2014) \\
\hline $3 \mathrm{~B}$ & $113.8-139.8$ & $698.62-760.71$ & TGW (Y) & QTgw.cau-3B.1; QTgw.cau-3B.1 & E1/E4/E6/E8/E9/E11/C & Cui et al. (2014) \\
\hline \multirow[t]{3}{*}{$5 B$} & $6.0-28.4$ & $35.29-395.63$ & TGW (Y) & QTgw.cau-5B.2; QTgw.cau-5B.3 & E2/E3/E5/E6/E7/C & Cui et al. (2014) \\
\hline & & & GA (Y) & QGa.cau-5B.2 & E6/E7/E11/C & \\
\hline & & & GC (Y) & $\begin{array}{l}Q G \text { c.cau-5B.1; QGc.cau-5B.3; } \\
\text { QGc.cau-5B.5 }\end{array}$ & E6/E7/E8/E9/E11/C & \\
\hline \multirow[t]{8}{*}{$6 \mathrm{~A}$} & $62.5-94.2$ & $52.40-585.43$ & GL (Y) & $\begin{array}{l}\text { QGl.cau-5B.1; QGl.cau-5B.3; } \\
\text { QGl.cau-5B.4 }\end{array}$ & $\begin{array}{l}\text { E5/E6/E7/E8/E9/E10/ } \\
\text { E11/C }\end{array}$ & Cui et al. (2014) \\
\hline & & & GNS (J) & $\begin{array}{l}\text { QGns.cau-6A.2; QGns.cau- } \\
\text { 6A.3;QGns.cau-6A.4 }\end{array}$ & $\begin{array}{l}\text { E1/E2/E3/E4/E5/E6/E7/ } \\
\text { E8/E9/E10/E11/C }\end{array}$ & Jia et al. (2013) \\
\hline & & & GNS (J) & $\begin{array}{l}\text { QGns.cau-6A.2; QGns.cau- } \\
\text { 6A.3;QGns.cau-6A.4 }\end{array}$ & $\begin{array}{l}\text { E1/E2/E3/E4/E5/E6/E7/ } \\
\text { E8/E9/E10/E11/C }\end{array}$ & Jia et al. (2013) \\
\hline & & & TGW (Y) & $\begin{array}{l}\text { QTgw.cau-6A.1; } ; \text { QTgw.cau- } \\
\text { 6A.2;QTgw.cau-6A.3 }\end{array}$ & $\begin{array}{l}\text { E1/E2/E3/E4/E5/E6/E7/ } \\
\text { E9/E10/E11/C }\end{array}$ & $\begin{array}{l}\text { Cui et al. (2014); } \\
\text { Simmonds et al. } \\
\text { (2014) }\end{array}$ \\
\hline & & & GA (Y) & $\begin{array}{l}\text { QGa.cau-6A.1; QGa.cau-6A.2; } \\
\text { QGa.cau-6A.3 }\end{array}$ & $\begin{array}{l}\text { E5/E6/E7/E8/E9/E10/ } \\
\text { E11/C }\end{array}$ & \\
\hline & & & GC (Y) & $\begin{array}{l}\text { QGc.cau-6A.2; } Q G \text { c.cau-6A.3; } \\
\text { QGc.cau-6A.4 }\end{array}$ & E5/E6/E7/E8/E9/E11/C & \\
\hline & & & GL (Y) & QGl.cau-6A.1; QGl.cau-6A.2 & E5/E6/E7/E8/E9/E11/C & \\
\hline & & & GW (Y) & $\begin{array}{l}Q G w . c a u-6 A .1 ; Q G w . c a u-6 A .2 ; \\
\text { QGw.cau-6A.3 }\end{array}$ & E5/E6/E7/E8/E9/E11/C & Cui et al. (2014) \\
\hline $6 \mathrm{~B}$ & $1.3-31.9$ & & GL (Y) & QGl.cau-6B.2; QGl.cau-6B.3 & E5/E6/E9/E11/C & \\
\hline \multirow[t]{2}{*}{$7 \mathrm{~A}$} & $53.9-85.2$ & $76.98-275.92$ & GNS (J) & QGns.cau-7A.2; QGns.cau-7A.3 & E3/E5/E6/E7/E9/E10/C & Kumar et al. (2007) \\
\hline & & & GL (Y) & QGl.cau-7A.1 & $\mathrm{E} 5 / \mathrm{E} 7 / \mathrm{E} 11 / \mathrm{C}$ & \\
\hline
\end{tabular}

${ }^{a}$ Additional details regarding the SNP markers within each QTL region can be found in Zhai et al. (2016)

${ }^{\mathrm{b}}$ The corresponding physical intervals $(\mathrm{Mb})$ of the QTL regions on chromosomes $2 \mathrm{~B}, 3 \mathrm{~A}, 3 \mathrm{~B}, 5 \mathrm{~B}, 6 \mathrm{~A}$ or $7 \mathrm{~A}$ were obtained by blasting the flanking sequences of SNP markers to the Chinese Spring RefSeq v1.0 sequence (Supplementary Table S9)

${ }^{\mathrm{c}}$ The traits include thousand grain weight (TGW), grain number per spike (GNS), grain surface area (GA), grain circumference (GC), grain length (GL) and grain width $(\mathrm{GW})$. The letters within the brackets indicate the origin of the increasing alleles, with ' $\mathrm{Y}$ ' and ' $\mathrm{J}$ ' representing Y8679 and J411, respectively

${ }^{\mathrm{d}}$ QTLs shown in bold are stable QTLs that were detected in $\geq 3$ individual environments and in the analysis of BLUPs

${ }^{\mathrm{e}} \mathrm{C}$ indicates the combined QTL analysis based on BLUP values 

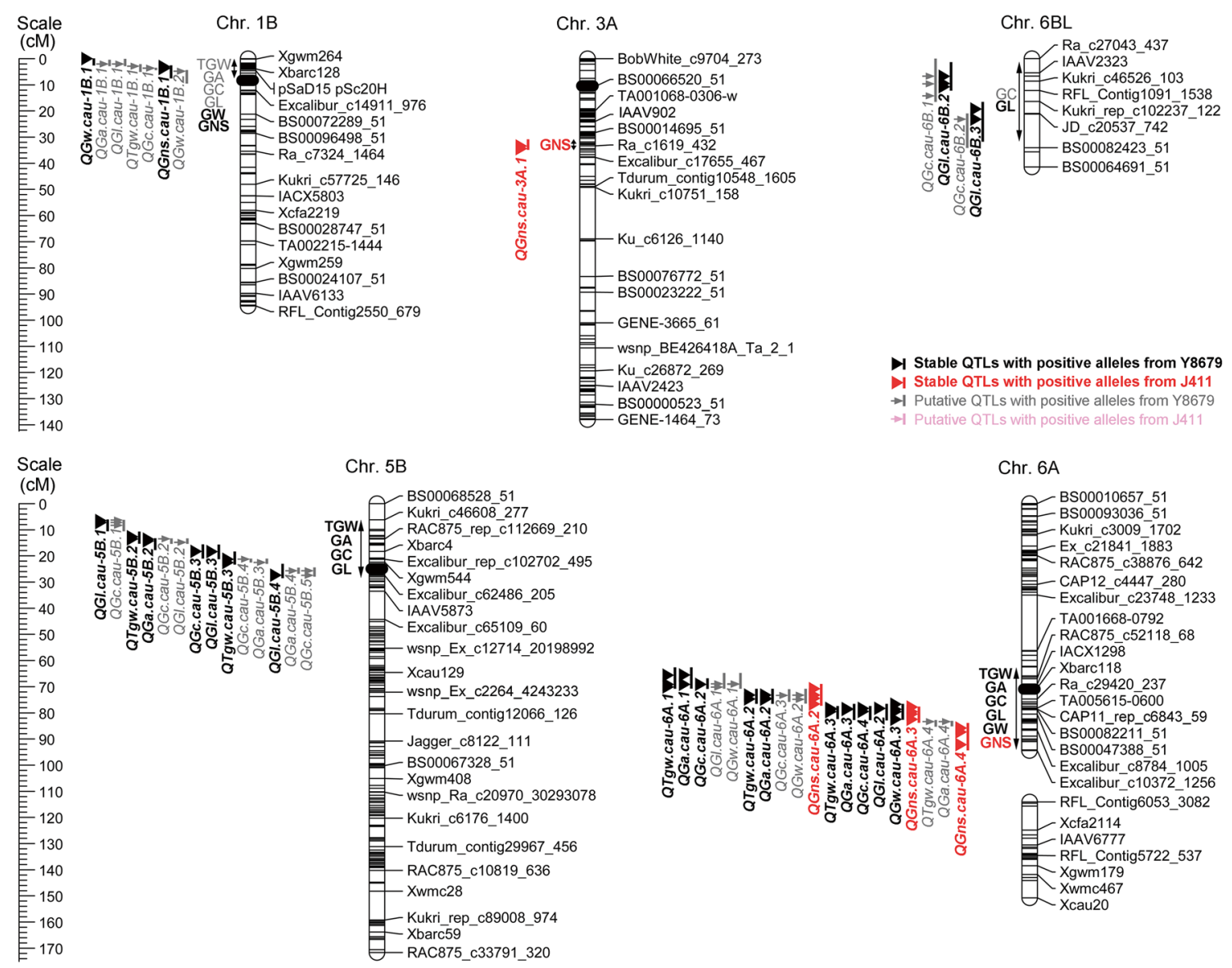

Stable QTLs with positive alleles from Y8679 S Stable QTLs with positive alleles from J411 $\rightarrow$ Putative QTLs with positive alleles from Y8679

- BSO0000523_51 $\rightarrow 1$ Putative QTL wi
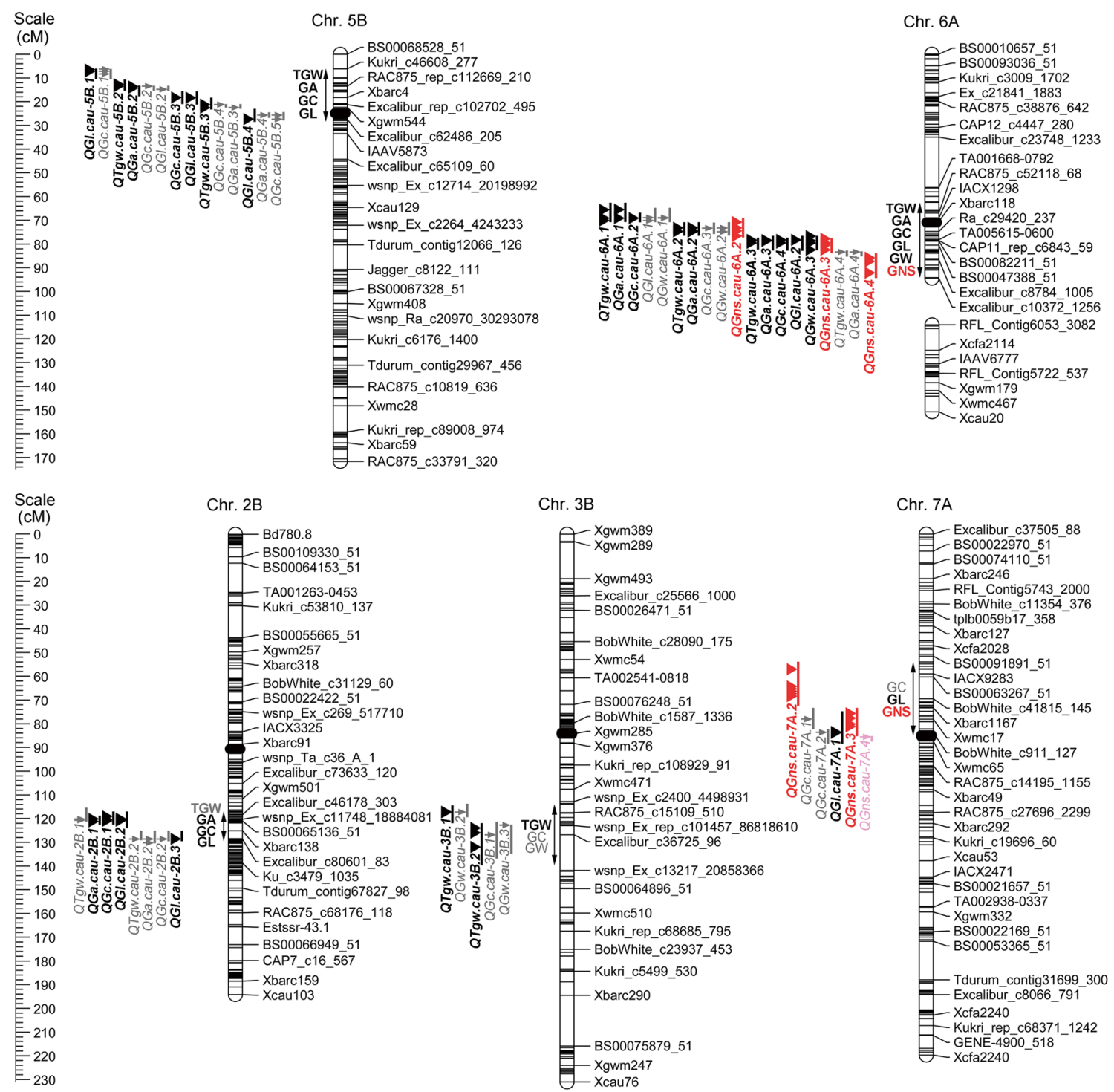

Fig. 1 Chromosomal locations of eight genomic regions associated with TGW, GNS, and four grain morphometric parameters in the Y8679/J411 population. Three centiMorgan (cM) scales are shown on the left. Information regarding the omitted SNP and SSR markers (represented as horizontal black lines) can be accessed in Zhai et al. (2016). Solid black ellipses indicate the centromeres. Double-headed arrows specify the interval of a genomic region harboring QTLs or

QTL clusters. Vertical bars represent the LOD-2 confidence intervals of each QTL, with triangles or arrows indicating the QTL peaks in individual environments. Stable QTLs are shown in bold black or bold red, with superior alleles coming from Y8679 or J411, respectively. Putative QTLs are displayed in gray and pink, with Y8679 or J411 contributing the increasing alleles, respectively 
1B co-localized with the 1RS/1BL translocation, which is present in Y8679 and has a strong positive effect on spikelet number per spike (Zhai et al. 2016). QGns.cau-1B.1 was the only GNS QTL at which Y8679 contributed the increasing allele. In detected environments, QGns.cau-1B.1 explained $8.49-15.25 \%$ of the total variation of GNS. The region on chromosome 3A contained another stable QTL for GNS (QGns.cau-3A.1) with a relatively minor effect. The region on chromosome 7A contained two adjacent major GNS QTLs (QGns.cau-7A.2 and QGns.cau-7A.3) that together explained $29.63 \%$ of the observed variation of GNS. For both regions on chromosomes $3 \mathrm{~A}$ and 7A, the alleles from J411 were found to have significant positive effects on GNS. The RILs carrying positive alleles at all three of the stable QTL regions for GNS (YJJ) exhibited significantly higher GNS (by 4.87 grains, $P<0.0001$ ) and GWS (by $0.28 \mathrm{~g}, P<0.0001$ ) (Fig. 2a) than those possessing the three opposite alleles (JYY). The two groups did not differ significantly $(P=0.36)$ in TGW.

The stable QTL regions on chromosomes $3 \mathrm{~B}$ and $5 \mathrm{~B}$ mainly influenced TGW, with the superior alleles coming from Y8679 (Fig. 1; Table 2). In the analysis of BLUPs, QTgw.cau-3B.1 and QTgw.cau-3B.2 explained 7.35 and $6.44 \%$ of the variation observed for TGW, respectively. A QTL for GW (QGw.cau-3B.2) coincided with QTgw.cau$3 B .1$, suggesting possible pleiotropy at this locus. The region on chromosome 5B covered two stable QTLs for TGW, i.e., QTgw.cau-5B.2 and QTgw.cau-5B.3, which together explained $12.36 \%$ of the total variation of TGW. This region also possessed stable QTLs for GA (QGa.cau-5B.2), GC (QGc.cau-5B.3), and GL (QGl.cau-5B.3 and QGl.cau-5B.4), indicating that the positive effect on TGW was most likely conferred by increasing GL. The RILs carrying positive alleles in both the $3 \mathrm{~B}$ and $5 \mathrm{~B}$ regions (YY) had higher TGW (by $4.53 \mathrm{~g}, P<0.0001$ ) and GWS (by $0.12 \mathrm{~g}, P<0.001$ ) and slightly lower GNS (by 0.12 grains, $P<0.05$ ) than those possessing the two opposite alleles (JJ) (Fig. 2b).

In the stable QTL region on chromosome 6A, the allele from Y8679 increased TGW but decreased GNS (Fig. 3a; Table 2). This region contained three major QTLs for TGW, i.e., QTgw.cau-6A.1, QTgw.cau-6A.2, and QTgw.cau-6A.3,
(A)

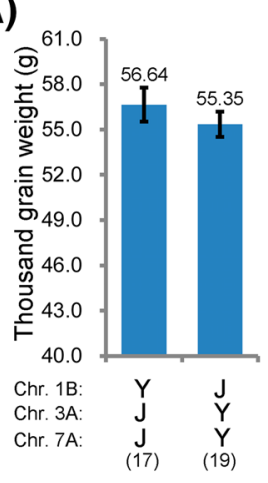

(C)

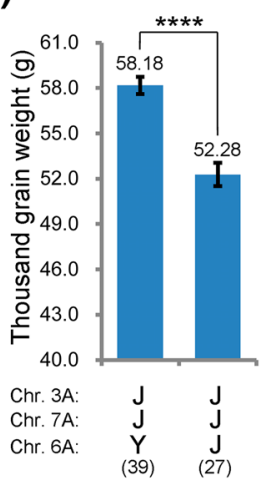

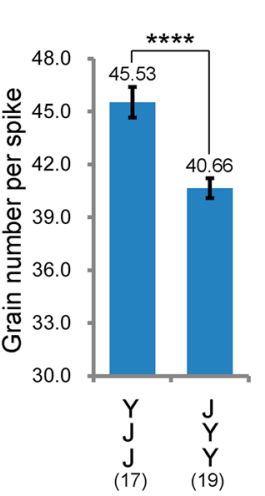

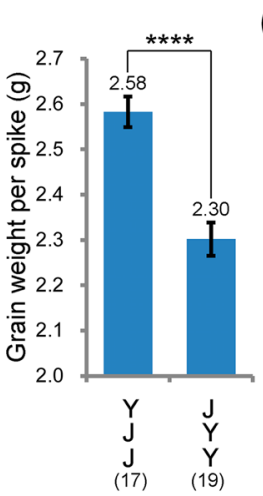

(B)
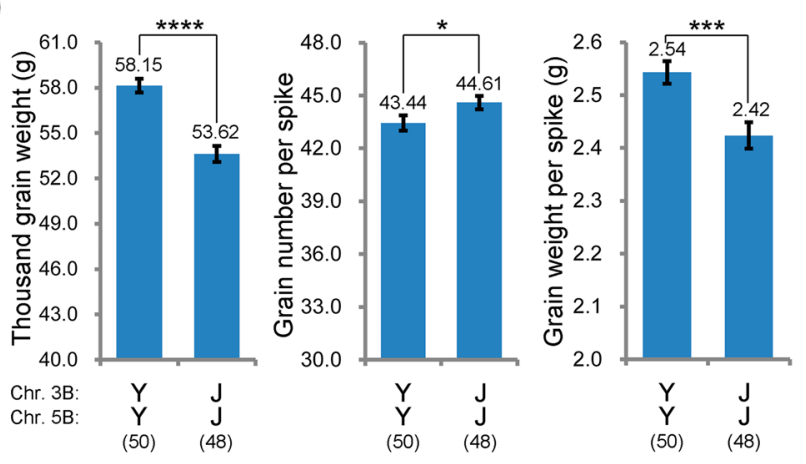

(D)
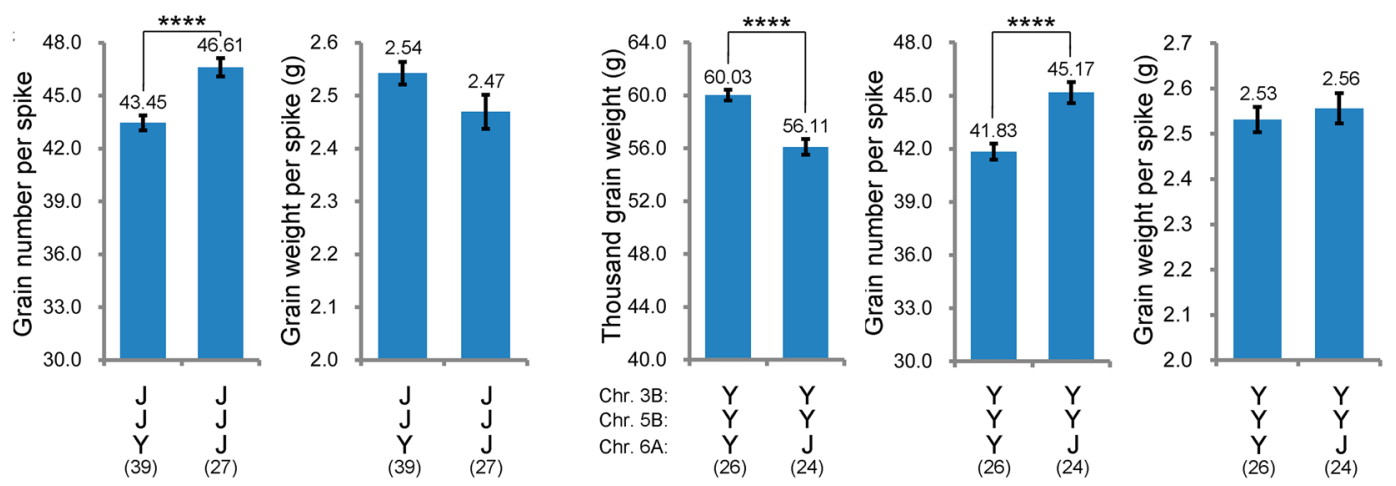

Fig. 2 Pyramiding effects of several stable QTL regions on chromosomes $1 \mathrm{~B}, 3 \mathrm{~A}, 3 \mathrm{~B}, 5 \mathrm{~B}, 6 \mathrm{~A}$, or $7 \mathrm{~A}$. The phenotypic data used here were the BLUP estimates of each recombinant inbred line (RIL) across 11 environments. The genotypic data used here were collected from the allelic information at specific markers within the stable QTL regions as follows: $p S c 20 H$ (Chr. 1B), Ra_c1619_432 (Chr. 3A), RAC875_c15109_510 (Chr. 3B), Excalibur_rep_c102702_495

(Chr. 5B), Ra_c29420_237 (Chr. 6A), and BobWhite_c41815_145 (Chr. 7A). 'Y' and ' $\mathrm{J}$ ' indicate alleles contributed by $\mathrm{Y} 8 \overline{6} 79$ and $\mathrm{J} 411$, respectively. For a specific pyramiding pattern, the number of RILs is shown in the bracket, and the mean value of these RILs ( \pm SE) is shown in a histogram. *, ***, and $* * * *$ indicate significant differences at the 0.05, 0.001, and 0.0001 levels (Student's $t$ test), respectively 
which together explained $49.20 \%$ of the total variation of TGW. Moreover, several major QTLs for GA (QGa.cau6A.1, QGa.cau-6A.2, and QGa.cau-6A.3) and GC (QGc. cau-6A.2 and QGc.cau-6A.4) were also detected in this region. Together, these QTLs explained 50.14 and 27.08\% of the total variation of GA and GC, respectively. Y8679 contributed increasing alleles for all of these loci. Two major QTLs for GNS (QGns.cau-6A.2 and QGns.cau-6A.3) with superior alleles coming from $\mathrm{J} 411$ were also detected in this region. Together, these QTLs explained $38.60 \%$ of the observed GNS variation. Even in the presence of high-GNS alleles on chromosomes $3 \mathrm{~A}$ and 7A or high-TGW alleles on chromosomes 3B and 5B, the RILs with Y8679 allele at the 6 A region had higher TGW (by $3.92-5.90 \mathrm{~g}, P<0.0001$ ) and lower GNS (by 3.16-3.24 grains, $P<0.0001$ ) than those possessing $\mathrm{J} 411$ allele (Fig. 2c, d).

For GL, there were also additional stable QTLs in regions on chromosomes $2 \mathrm{~B}$ and $6 \mathrm{~B}$, but these QTLs did not significantly influence TGW (Figs. 1,2). The region on chromosome 2B harbored the two most stable QTLs for GL, i.e., $Q G$ Gl.cau-2B.2 and QGl.cau-2B.3, which were found in nearly all evaluated environments (Supplementary Table S8). These two loci together explained $19.02 \%$ of the detected GL variation. QGl.cau-2B.2 co-localized with two stable QTLs for GA (QGa.cau-2B.1) and GC (QGc.cau$2 B .1$ ), which explained 4.92 and $8.81 \%$ of the total variation of GA and GC, respectively. The region on chromosome 6B contained two QTLs for GL, i.e., $Q G l . c a u-6 B .2$ and $Q G l$. cau-6B.3, which together explained $13.81 \%$ of the observed GL variation.

\section{Use of NILs to verify and precisely map a QTL on chromosome $6 \mathrm{~A}$}

RIL186 was selected from the population, because this RIL exhibited residual heterozygosity at locus Xbarc 118. Two of its selfed progeny, which possessed alternative haplotypes and were homozygous, were genotyped using the Affymetrix wheat $660 \mathrm{~K}$ SNP array (http://wheat.pw.usda.gov/ggpages/ topics/

Wheat660_SNP_array_developed_by_CAAS.pdf). The results showed that they were $99.82 \%$ similar, only differing in 1094 SNPs (Supplementary Table S11). Of these 1094 polymorphic SNPs, 895 were located in an interval between 28.22 and $445.78 \mathrm{Mb}$ on chromosome $6 \mathrm{~A}$. The flanking sequences of these 895 SNP markers and their physical locations in the Chinese Spring RefSeq v1.0 sequence are listed in Supplementary Table S12. Considering that the corresponding physical interval of the $6 \mathrm{~A}$ region spanned from 52.40 to $585.43 \mathrm{Mb}$, we chose to focus on the region from 52.40 to $445.78 \mathrm{Mb}$ (Table 2), i.e., an 8.908 cM interval between wsnp_BE490604A_Ta_2_1 and
wsnp_RFL_Contig1340_448996 and designated as QTgw/ Gns.cau-6A, for the development of NILs (Fig. 3a, b).

Fourteen co-dominant polymorphic SSR markers (Supplementary Table S6) were developed using a collinearitybased strategy and used to genotype the $\mathrm{F}_{9: 10}$ family with 163 individuals derived from RIL186. The resultant genetic linkage map spanned $12.18 \mathrm{cM}$ in length, covering an interval from 31.39 to $237.76 \mathrm{Mb}$ in the Chinese Spring RefSeq v1.0 sequence (Supplementary Table S13). Based on genotype of these markers, four sets of NIL pairs (NIL1, NIL2, NIL3, and NIL4) with overlapping recombinant segments were developed (Fig. 3c). The first NIL pair, with no recombination in the concern region, was used to verify the effects of $Q T g w /$ Gns.cau-6A.

Across six replicated field trials, the TGW of NIL1Y8679 (with Y8679 haplotype) was from 6.68 to $11.90 \%$ (mean $8.33 \%, P<0.001$ ) higher than that of NIL1-J411 (Fig. 4; Supplementary Table S14). This variation in TGW was associated with average differences of $4.95,1.87,1.19$, and $3.30 \%(P<0.001)$ in GA, GC, GL, and GW, respectively, with NIL1-Y8679 having the higher values. A difference in GNS (mean $3.05 \%, P<0.001$ ) was also observed, with NIL1-Y8679 exhibiting the lower value. This effect varied across six trials with NIL1-Y8679 associated with significant GNS reductions $(P<0.05)$ from 2.71 to $8.20 \%$ in five of them. Overall, NIL1-Y8679 had 3.35\% higher GWS $(P<0.001)$ and $4.29 \%$ higher GWP $(P<0.001)$ than NIL1J411. In most trials, no significant differences were observed for tiller number (Supplementary Table S14). Collectively, these results demonstrate that the Y8679 haplotype in $Q T g w /$ Gns.cau-6A has a consistent positive effect on GWS and GWP, which is conferred by a large positive effect on TGW, but counterbalanced by a small negative effect on GNS.

Subsequently, we used NIL2, NIL3, and NIL4 to map the high-TGW, low-GNS region into a smaller genetic interval. When these NIL pairs were evaluated for TGW and GNS in three field trials, significant differences in TGW and GNS were found only between the members of the NIL4 pair (Fig. 3d), but not between the members of the NIL2 or NIL3 pairs. Based on these results, QTgw/Gns. cau-6A was narrowed to an interval of $208.80 \mathrm{Mb}$, which is flanked by marker $6 A S-165(236.98 \mathrm{Mb})$ and the distal margin of the region of interest $(445.78 \mathrm{Mb}$; Supplementary Table S12). This represents a genetic interval shorter than $0.538 \mathrm{cM}$ on the Y8679/J411 map, a segment flanked by BS00027313_51 (70.873 cM; $151.49 \mathrm{Mb})$ and wsnp_RFL_ Contig1340_448996 (71.411 cM; 445.25 Mb) (Fig. 3a).

To estimate the additive and dominance effects of $Q T g w /$ Gns.cau-6A, we evaluated the $163 \mathrm{~F}_{10: 11}$ families for TGW, GNS, and GWS in one field trial (Beijing, 2014-15 crop season) in three replicates. Eighty segregating families and 36-41 homozygous families with alternative haplotypes 


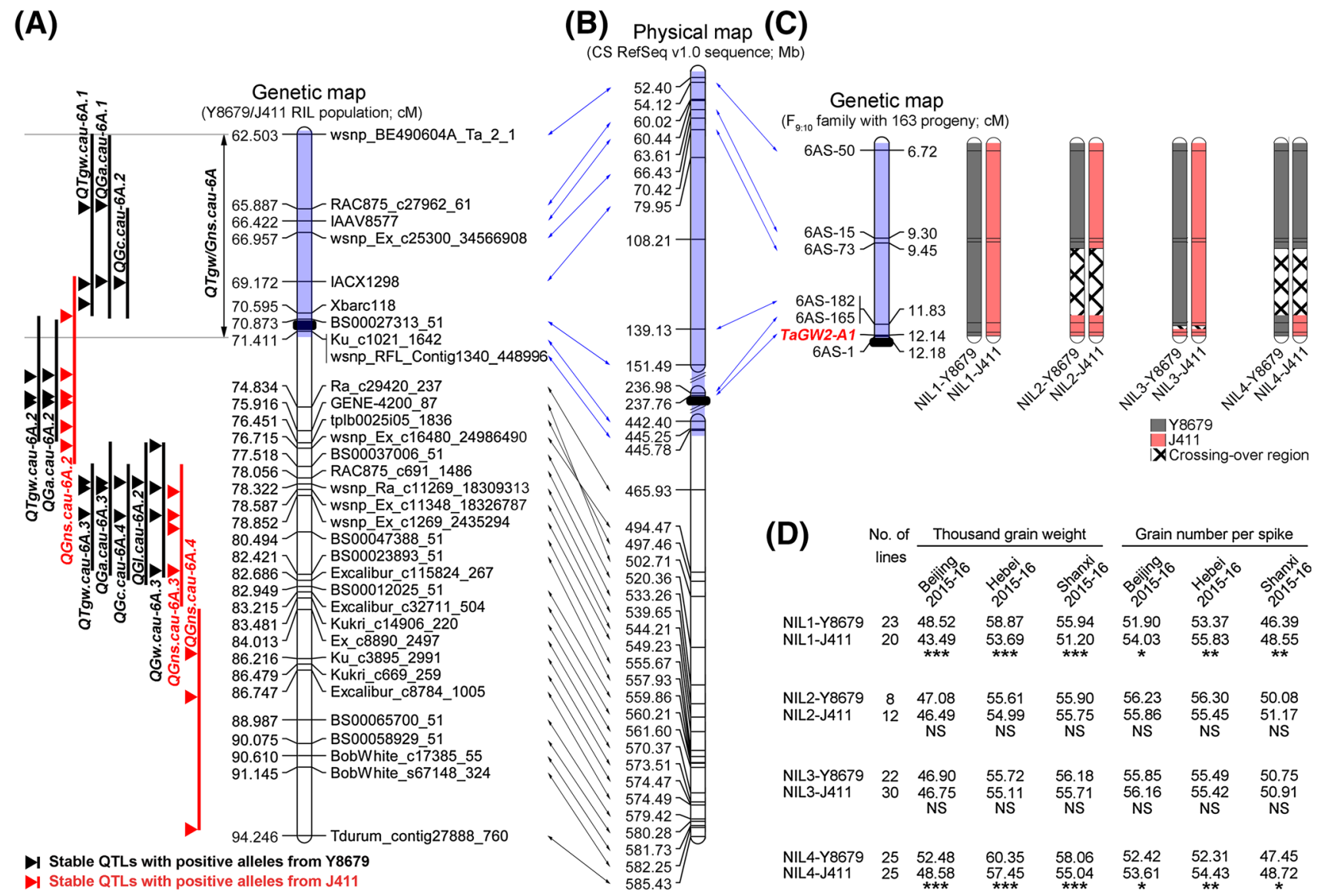

Fig. 3 Fine mapping of $Q T g w / G n s . c a u-6 A$. a Distributions of major QTLs for TGW, GNS, and four grain morphometric parameters within the stable QTL region on chromosome 6A. The interval colored in light blue represents $Q T g w / G n s . c a u-6 A$, which possessed whole QTgw.cau-6A.1 and partial QGns.cau-6A.2. Descriptions regarding the QTL confidence intervals and the peak regions are consistent with those introduced in Fig. 1. The solid black ellipse indicates the centromere. b Corresponding physical interval of the stable

across the interval from $6 A S$ - 165 to $6 A S$ - 1 were used for variation analysis. The Y8679 haplotype of $Q T g w / G n s . c a u-$ $6 A$ showed overdominance $(d / a=1.76)$ for higher TGW, with the mean values of segregating families $(43.51 \mathrm{~g})$ and Y8679-type homozygous families (43.75 g) being significantly higher $(P<0.001)$ than that of J411-type homozygous families (39.78 g). Likewisely, the J411 haplotype of QTgw/Gns.cau-6A exhibited overdominance $(d / a=2.16)$ for higher GNS, with the segregating families and J411-type homozygous families bearing 2.80 and 2.69 more grains $(P<0.001)$ than the Y8679-type homozygous families, respectively. We also observed an overdominance effect $(d / a=11.04)$ of $Q T g w / G n s . c a u-6 A$ on spike yield, with the mean GWS values of segregating families being $0.11-0.16 \mathrm{~g}$ higher $(P<0.01)$ than those of the two types of homozygous families (Table 3).
QTL region on chromosome 6A in the Chinese Spring RefSeq v1.0 sequence. The solid black ellipse indicates the centromere. c Graphical genotypes of four NIL pairs (derived from RIL186) with overlapping recombinant segments. The solid black ellipse indicates the centromere. d Performance of the members of the four NIL pairs in three field trials. *, **, and $* * *$ indicate significant differences at the 0.05 , 0.01 , and 0.001 levels (Student's $t$ test), respectively

\section{Isolation of candidate gene TaGW2-A1}

$T a G W 2-A 1$, a wheat homolog of rice $O s G W 2$ (Song et al. 2007), was previously reported to negatively affect TGW in wheat (Du et al. 2016; Simmonds et al. 2016; Yang et al. 2012). It was mapped to the short arm of chromosome 6A, near the centromere ( $\mathrm{Su}$ et al. 2011). As this position corresponds with that of $Q T g w / G n s . c a u-6 A$, we obtained the complete coding sequence and the promoter sequence (about $2.0 \mathrm{~kb}$ upstream from ATG) for TaGW2-A1 from Y8679 to J411, and analyzed their polymorphisms. No differences were observed in the coding sequence, but a 114-bp Insertion/Deletion (InDel) was detected in the promoter region ( -230 to -117 -bp upstream from ATG; Fig. 5a). No other variations were detected in the rest of the promoter sequence. The allele from Y8679 has the 114-bp deletion as 


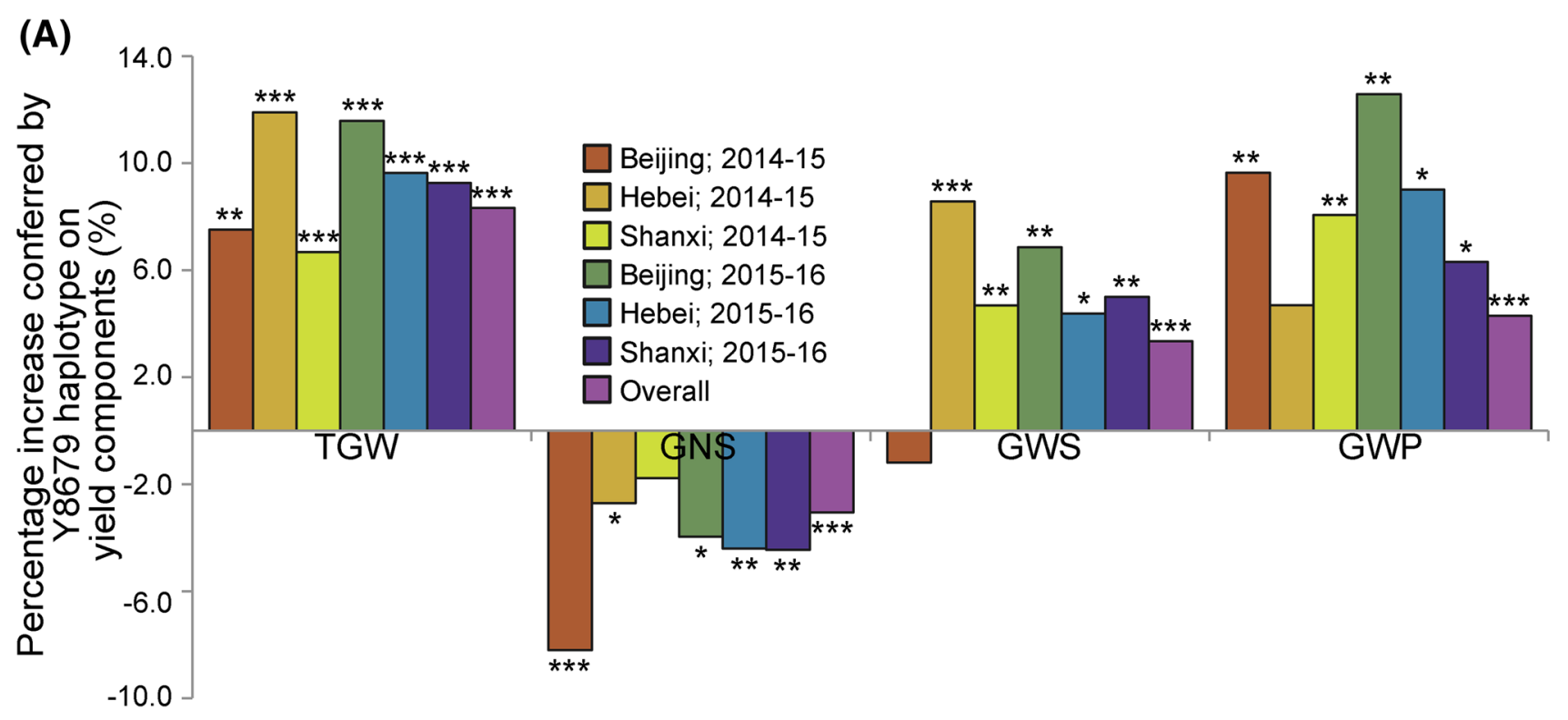

(B)

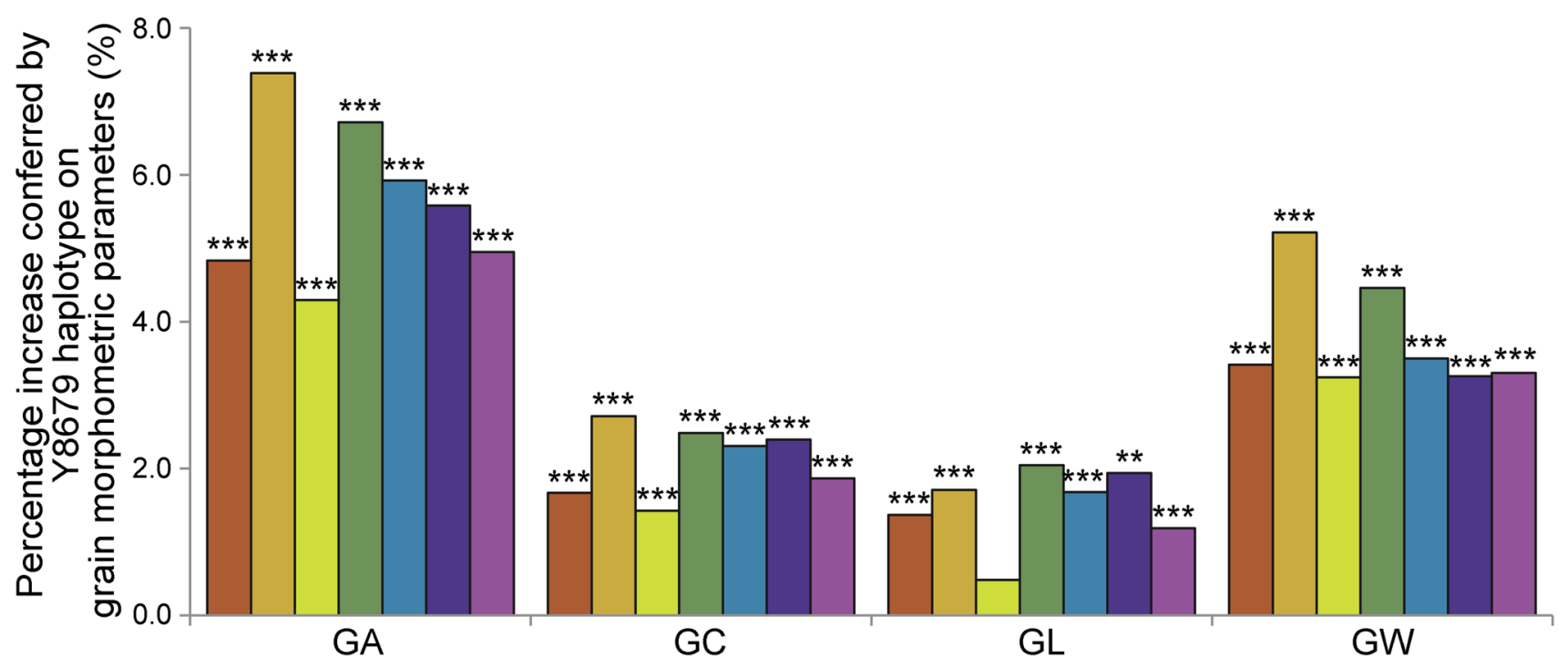

Fig. 4 Phenotypic comparisons of NIL1-Y8679 and NIL1-J411 in six field trials. Percentage increase conferred by the Y8679 haplotype on a yield components and $\mathbf{b}$ grain morphometric parameters.
Significant differences are indicated by $* P<0.05$, $* * P<0.01$, and $* * * P<0.001$ (Student's $t$ test). 'Overall' represents the BLUP values estimated across six evaluated environments

and a 221-bp 3'UTR. The transcription start site (- 176-bp upstream from ATG) was located within the 114-bp InDel (Fig. 5a). We fused the TaGW2-Al promoter sequences from Y8679 (1121 bp) and J411 (1235 bp) with the GUS reporter gene (Supplementary Fig. S3), respectively, to assess the effects of the 114-bp InDel on promoter activity. The GUS activity conferred by Y8679-pTaGW2-A1::GUS was 76.83\% lower $(P<0.001)$ than that by J11-pTaGW2-A1::GUS, but still $48.96 \%$ higher $(P<0.01)$ than that detected for the empty vector control (Fig. 5b). In the immature grains (11 days after pollination), the expression level of 
Table 3 Estimation of the additive and dominance effects of $Q T g w / G n s . c a u-6 A$ on TGW, GNS, and GWS using $163 \mathrm{~F}_{10: 11}$ families

\begin{tabular}{llll}
\hline Families & TGW & GNS & GWS \\
\hline Y8679-type homozygous families & $43.75 \pm 0.50^{\mathrm{A}}$ & $41.36 \pm 0.59^{\mathrm{B}}$ & $1.82 \pm 0.04^{\mathrm{B}}$ \\
Segregating families & $43.51 \pm 0.28^{\mathrm{A}}$ & $44.16 \pm 0.33^{\mathrm{A}}$ & $1.93 \pm 0.02^{\mathrm{A}}$ \\
J411-type homozygous families & $39.78 \pm 0.30^{\mathrm{B}}$ & $44.05 \pm 0.35^{\mathrm{A}}$ & $1.77 \pm 0.02^{\mathrm{B}}$ \\
$a$ & 1.98 & 1.35 & 0.02 \\
$d$ & 3.49 & 2.91 & 0.26 \\
$d / a$ & 1.76 & 2.16 & 11.04 \\
\hline
\end{tabular}

The $163 \mathrm{~F}_{10: 11}$ families derived from RIL186 were evaluated at Beijing during the 2014-15 crop season with three replicates. Based on the genotypes of $6 A S-165$ and $6 A S-1,80$ segregating families, 41 Y8679type homozygous families and 36 J411-type homozygous families were selected and used for phenotypic variation analysis. $a$ and $d$ indicate the additive and dominance effects, respectively. $d / a$ indicates the degree of dominance. Multiple comparison was based on LSD method. Different letters $\left({ }^{\mathrm{A}}\right.$ and $\left.{ }^{\mathrm{B}}\right)$ are used to indicate the means $( \pm \mathrm{SE})$ that significantly differ $(P<0.01)$

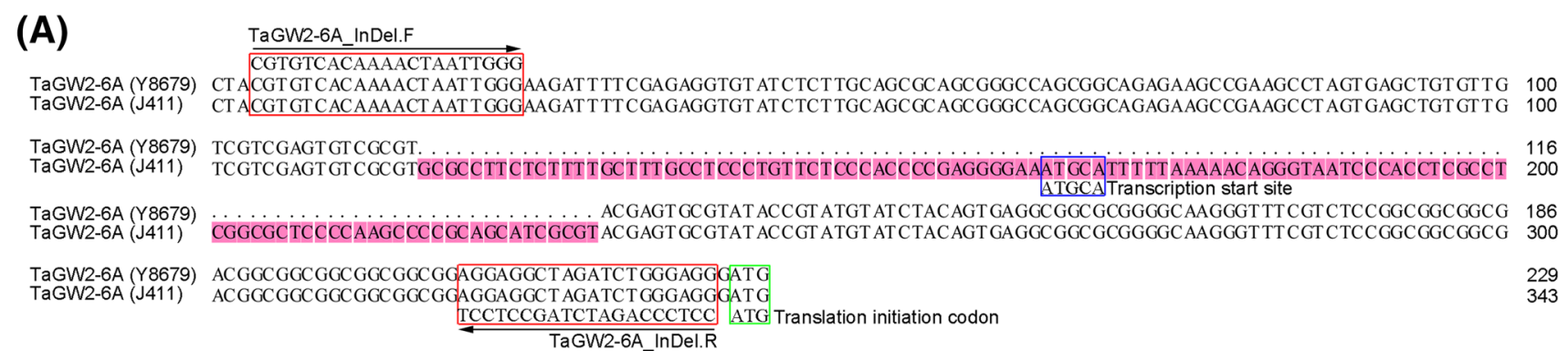

(B)

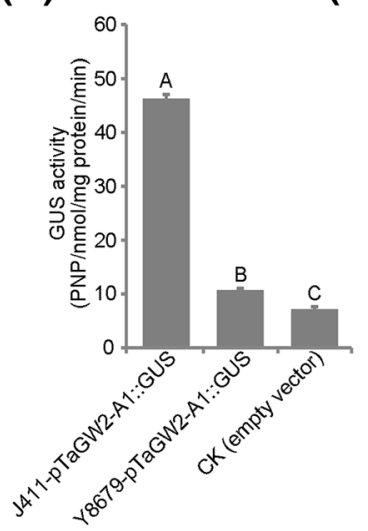

(C)

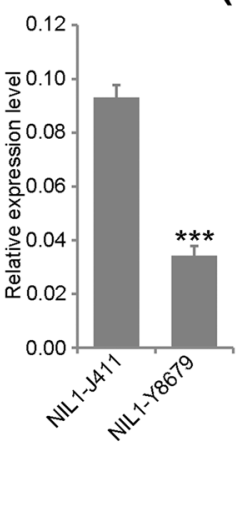

(D)

Hexaploid wheat species (AABBDD) Chinese modern cultivars: $702(3 ; 699)$

Chinese landraces: $96(0 ; 96)$

French varieties: $50(0 ; 50)$

Tetraploid wheat species (AABB) Triticum dicoccoides: $181(0 ; 181)$

Triticum durum: $33(0 ; 33)$

Triticum dicoccum: $24(0 ; 24)$

Diploid wheat species (AA)

Triticum urartu: $6(0 ; 6)$

Triticum boeoticum: $6(0 ; 6)$

Triticum monococcum: $15(0 ; 15)$
(E)

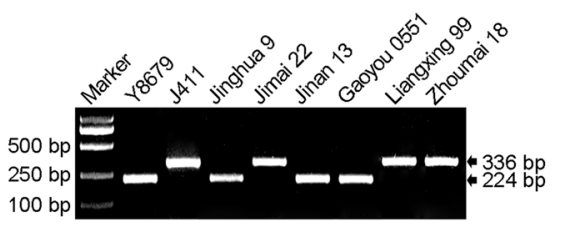

Fig. 5 Isolation of a novel and rare allele of TaGW2-A1. a 114-bp Insertion/Deletion (InDel) detected in the promoter region. Red rectangles specify the forward and reverse primers of TaGW2-A1_InDel. Blue and green rectangles indicate the transcription start site and the predicted translation initiation codon, respectively. b Comparison of the GUS expression level in different promoter-GUS constructs. Superscripts A, B, and C indicate the means ( \pm SE) that significantly differ at the 0.01 level (LSD). c Relative expression levels of TaGW2-A1 in immature grains (11 dap) of the NIL1 pair, with Actin

TaGW2-Al in NIL1-Y8679 was $63.27 \%$ lower $(P<0.001)$ than that in NIL1-J411 (Fig. 5c). Taken together, we propose that the 114-bp deletion reduces the promoter activity and expression of $T a G W 2-A 1$.

To further explore implications of our findings on wheat breeding, primer pair TaGW2-A1_InDel was used used as the endogenous control. The results of three biological replications showed similar trends. The values represent the means $( \pm \mathrm{SE})$ of three biological replicates. $* * *$ indicates significance at the 0.001 level (Student's $t$ test). d Frequency of the 114-bp deletion among 1113 wheat accessions with varying ploidy. The two numbers (from left to right) within the brackets indicate the number of accessions amplifying the 224-bp fragment and the 336-bp fragment, respectively. e PCR products of TaGW2-A1_InDel in several hexaploid wheat accessions

to amplify 848 hexaploid wheat accessions, including 798 Chinese varieties (96 landraces and 702 modern cultivars) and 50 French varieties (Fig. 5d; Supplementary Table S1). Nearly all accessions (99.6\%) amplified the wild-type 336bp J411 fragment, whereas only three (Jinan 13, Jinghua 9 and Gaoyou 0551) amplified the mutant type 222-bp Y8679 
fragment (Fig. 5e; Supplementary Fig. S7). Thus, at least within the panel evaluated, the favorable allele of $T a G W 2-A 1$ is very rare. Moreover, 238 tetraploid and 27 diploid wheat species were also screened for the 114-bp deletion (Fig. 5d; Supplementary Tables 2 and 3), but none of them carried the 224-bp fragment like Y8679, again suggesting a rare mutation event leading to the 114-bp deletion.

\section{Discussion}

\section{Five genomic regions exhibit negligible TGW-GNS trade-offs}

TGW and GNS are two primary yet negatively correlated determinants of spike yield in wheat (Jia et al. 2013; Shukla et al. 2015). Information about trade-offs between the two traits can help to improve the efficiency of wheat breeding. Here, we report five genomic regions on chromosomes 1B, $3 \mathrm{~A}, 3 \mathrm{~B}, 5 \mathrm{~B}$, or 7A exhibiting negligible TGW-GNS tradeoffs. These represent attractive targets for marker-assisted selection to enhance grain size and/or grain number. The region on chromosome $1 \mathrm{~B}$ can be attributed to the $1 \mathrm{RS} / 1 \mathrm{BL}$ translocation (carried by Y8679). It increased GNS, corresponding to our previous finding that 1RS/1BL translocation enhanced spikelet number per spike across all evaluated trials (Zhai et al. 2016). In the regions on chromosomes $3 \mathrm{~A}$ and $7 \mathrm{~A}$, alleles from J411 increased the grain number without significantly reducing grain weight. The $3 \mathrm{~A}$ region is probably within the deletion bin 3AL5-0.78-1.00, which has previously been reported to harbor QTLs for grain yield, GNS, spikelet number per spike, test weight, TGW, and heading time (Ali et al. 2011; Cui et al. 2014; Huang et al. 2004; Zhai et al. 2016). Similarly, the region detected on chromosome 7A has previously been reported to affect GNS, spikelet number per spike and grain yield (Kumar et al. 2007; Ma et al. 2007; Reif et al. 2011; Wu et al. 2012). In the regions on chromosomes 3B and 5B, alleles from Y8679 increased TGW without significantly reducing GNS. These two regions coincide with several previously reported QTLs for TGW and grain yield (Cui et al. 2014; Reif et al. 2011; Sun et al. 2009). Notably, the region on chromosome 3B has also been shown to enlarge grain size through enhancing grain filling rate in the Heshangmai/Y8679 population, which shares a common parent with the population used in this study (Wang et al. 2009).

\section{Analysis of the QTLs on chromosome 6A provides new insight into TGW-GNS trade-off}

In recent years, evidence is accumulating that robust QTLs for TGW (Cui et al. 2016; Mir et al. 2012; Snape et al. 2007; Zanke et al. 2015) and GNS (Jia et al. 2013; Yuan et al.
2012) exist in the pericentromeric region of chromosome 6A. Here, we detected stable major QTLs for TGW ( $Q T g w$. cau-6A.1, QTgw.cau-6A.2 and QTgw.cau-6A.3) and GNS (QGns.cau-6A.2, and QGns.cau-6A.3) in this region. The two QTL clusters on chromosome 6A for TGW and GNS were both linked in the coupling phase, but with superior alleles coming from opposite parents. Hence, selection for the higher TGW haplotype would inevitably be accompanied by a reduction of GNS, and vice versa. This is a typical selection trade-off problem for higher TGW versus higher GNS. Importantly, by evaluating a pair of NILs in several field trials, we demonstrated that $Q T g w / G n s . c a u-$ 6A (covering whole QTgw.cau-6A.1 and partial QGns.cau6A.2) affected both TGW and GNS in an opposite manner. Through further mapping with another three pairs of NILs, we narrowed $Q T g w / G n s$.cau-6A to a small genetic interval shorter than $0.538 \mathrm{cM}$. Consequently, QTgw/Gns.cau- $6 A$ and the NIL pairs developed here may be a perfect target and good materials, respectively, for further studying the molecular genetic basis of TGW-GNS trade-off in common wheat.

From a practical point of view, it is worthy to point out that when pyramiding the QTLs identified here, it seems advisable to select the Y8679 haplotype in the presence of major QTLs for GNS on chromosomes 3A and 7A (Fig. 2c), or the J411 haplotype in the presence of major QTLs for TGW on chromosomes 3B and 7B (Fig. 2d).

\section{A novel mutation of TaGW2-A1 is associated with increased TGW in wheat}

In rice, $O s G W 2$ encodes a RING-type protein with E3 ubiquitin ligase activity that negatively regulates the grain weight and width (Song et al. 2007). Its wheat homolog on the short arm of chromosome 6A ( $T a G W 2-A 1)$ locates in a region near the centromere (Su et al. 2011), in which major QTLs for TGW have been mapped (Cui et al. 2016; Simmonds et al. 2014; Zanke et al. 2015). Several reports (Su et al. 2011; Zhang et al. 2013, Jaiswal et al. 2015) have focused on the roles of individual SNPs in the promoter region, but validations of their associations with TGW are still needed. Two mutations in the coding sequence have been reported to be associated with TGW: a frame-shift mutation in exon 8 (Du et al. 2016; Yang et al. 2012) and a splice acceptor site mutation in exon 5 (Simmonds et al. 2016). The effects of these coding sequence mutations have been tested in backcross derived isogenic lines, providing sound evidence that $T a G W 2-A 1$, indeed, influences TGW in tetraploid and hexaploid wheat.

In the present study, we located $T a G W 2-A 1$ within the finely mapped interval of $Q T g w / G n s . c a u-6 A$. Furthermore, a novel mutation of $T a G W 2-A 1$ with a 114-bp deletion in the promoter was isolated from the high-TGW parent Y8679. Deletion of the wild-type transcription start site reduced 
promoter activity and decreased expression of $T a G W 2-A 1$, which might, in turn, enhance TGW according to the previous finding that $T a G W 2-A 1$ is a negative regulator of grain weight (Du et al. 2016; Simmonds et al. 2016; Yang et al. 2012). In a germplasm survey of more than 800 hexaploid wheat accessions, the 114-bp deletion was detected only in Y8679 and three other cultivars: Jinan 13, Jinghua 9, and Gaoyou 0551 (Fig. 5e). These four cultivars could be useful as donors for higher TGW in wheat breeding programs. Due to the incomplete pedigree information (Supplementary Table S15), no clear relationship was observed in these accessions. Furthermore, this novel allele was not detected in tetraploid and diploid wheat species that were involved in the formation of hexaploid wheat (IWGSC 2014). Together, these data point to the possibility that the novel TaGW2$A l$ allele identified here may arise during or after the hexaploidization event that yielded hexaploid wheat.

\section{QTgw/Gns.cau-6A shows possible overdominance effect on spike yield}

Heterosis has been a main contributor to yield increase in many cereal crops (Fu et al. 2014). Despite extensive efforts in hybrid wheat breeding, mechanisms of wheat heterosis are largely unknown (Ni et al. 2013). Here, we provide the first example of overdominance effect on spike yield that conferred by a single locus in wheat. Data from a single year trial (Beijing; 2014-15) suggest that the combination of the Y8679 haplotype and the J411 haplotype of $Q T g w / G n s$. cau-6A in the heterozygotes will most likely lead to an overdominance effect on GWS. Using the NILs developed for QTgw/Gns.cau-6A, the consistency of this overdominance effect across environments will be further investigated in the future.

Previously, Bednarek et al. (2012) found that $T a G W 2$ knockdown through RNAi caused no significant alternation in GNS or in the number of spikelets per spike. Similarly, Hong et al. (2014) also found that TaGW2-RNAi had no significant impact on grain number per plant, and Simmonds et al. (2016) found that a G-to-A transition in the splice acceptor site of exon 5, which leads to mis-splicing in TaGW2-A1, had no significant impact on GNS or on the number of spikelets per spike. Thus, we tend to believe that the overdominance effect of $Q T g w / G n s . c a u-6 A$ on GWS was most likely caused by two or more different dominant genes linked in repulsion phase, with $T a G W 2-A 1$ for enlarged grain size, while the other for increased grain number. Dissection of $Q T g w / G n s . c a u-6 A$ into two distinct QTLs for TGW and GNS, respectively, may eliminate linkage drag, but it will be a challenging task, since $Q T g w / G n s$.cau- $6 A$ is located near the centromere. Considering the low recombination frequency of the pericentromeric region, utilization of the overdominance effect on GWS in the form of heterozygotes seems to be a feasible way to combine higher TGW and higher GNS phenotypes.

Author contribution statement $\mathrm{ZN}$ and QS conceived the project; SX developed the RIL population; HZ, ZF, and JL performed phenotyping of the RIL population under 11 environments; XD, YS, HZ, and LS performed cloning and expressional analyses of TaGW2-A1. HZ and ZQ performed marker development of the QTL region of interest; HZ developed the near isogenic lines; HZ, LL, and JL performed phenotyping of the near isogenic lines; HP, ZH, YY, and MX assisted in revising the manuscript; $\mathrm{HZ}$ and $\mathrm{ZN}$ analyzed experimental results and wrote the manuscript.

Acknowledgements This work was financially supported by the National Natural Science Foundation of China (Grant no. 91435204), the National Key Research and Development Program of China (Grant nos. 2016YFD0100402 and 2016YFD0100801) and China Agriculture Research System (Grant no. CARS-3-1-10). The authors would like to thank Professor Chaojie Xie (China Agricultural University), Professor Weining Song (Northwest A\&F University), Professor Dongfa Sun (Huazhong Agricultural University), and Professor Yuming Wei (Sichuan Agricultural University) for providing the tetraploid and diploid wheat accessions, the IWGSC for pre-publication access to the RefSeq v1.0 sequence, and Professor Daowen Wang and Dr. Abrar Hussain (Chinese Academy of Sciences) for critical review of the manuscript.

\section{Compliance with ethical standards}

Conflict of interest The authors declare that they have no conflict of interest.

Open Access This article is distributed under the terms of the Creative Commons Attribution 4.0 International License (http://creativecommons.org/licenses/by/4.0/), which permits unrestricted use, distribution, and reproduction in any medium, provided you give appropriate credit to the original author(s) and the source, provide a link to the Creative Commons license, and indicate if changes were made.

\section{References}

Ali ML, Baenziger PS, Al Ajlouni Z, Campbell BT, Gill KS, Eskridge KM, Mujeeb-Kazi A, Dweikat I (2011) Mapping QTL for agronomic traits on wheat chromosome $3 \mathrm{~A}$ and a comparison of recombinant inbred chromosome line populations. Crop Sci 51:553-566. https://doi.org/10.2135/cropsci2010.06.0359

Ashikari M, Sakakibara H, Lin SY, Yamamoto T, Takashi T, Nishimura A, Angeles ER, Qian Q, Kitano H, Matsuoka M (2005) Cytokinin oxidase regulates rice grain production. Science 309:741-745. https://doi.org/10.1126/science.1113373

Bednarek J, Boulaflous A, Girousse C, Ravel C, Tassy C, Barret P, Bouzidi MF, Mouzeyar S (2012) Down-regulation of the $T a G W 2$ gene by RNA interference results in decreased grain size and weight in wheat. J Exp Bot 63:5945-5955. https://doi. org/10.1093/jxb/ers249 
Bolser DM, Kerhornou A, Walts B, Kersey P (2015) Triticeae resources in ensembl plants. Plant Cell Physiol 56(1):e3 1-e3 11. https://doi.org/10.1093/pcp/pcu183

Churchill GA, Doerge RW (1994) Empirical threshold values for quantitative trait mapping. Genetics 138(3):963-971

Cui F, Zhao CH, Ding AM, Li J, Wang L, Li XF, Bao YG, Li JM, Wang HG (2014) Construction of an integrative linkage map and QTL mapping of grain yield-related traits using three related wheat RIL populations. Theor Appl Genet 127:659-675. https://doi.org/10.1007/s00122-013-2249-8

Cui F, Fan XL, Chen M, Zhang N, Zhao CH, Zhang W, Han J, Ji J, Zhao XQ, Yang LJ, Zhao ZW, Tong YP, Wang T, Li JM (2016) QTL detection for wheat kernel size and quality and the responses of these traits to low nitrogen stress. Theor Appl Genet 129:469-484. https://doi.org/10.1007/s00122-015-2641-7

Dong LL, Wang FM, Liu T, Dong ZY, Li AL, Jing RL, Mao L, Li YW, Liu X, Zhang KP, Wang DW (2014) Natural variation of TaGASR7-A1 affects grain length in common wheat under multiple cultivation conditions. Mol Breed 34:937-947. https://doi. org/10.1007/s11032-014-0087-2

Du DF, Gao X, Geng J, Li QY, Li LQ, Lv Q, Li XJ (2016) Identification of key proteins and networks related to grain development in wheat (Triticum aestivum L.) by comparative transcription and proteomic analysis of allelic variants in TaGW2-6A. Front. Plant Sci 7:922. https://doi.org/10.3389/fpls.2016.00922

Falconer DS, Mackay TFC (1996) Values and Means. Introduction to quantitative genetics, 4th edn. Addison Wesley Longman Limited, Harlow, pp 108-120

Fu D, Xiao M, Hayward A, Fu Y, Liu G, Jiang G, Zhang H (2014) Utilization of crop heterosis: a review. Euphytica 197:161-173. https://doi.org/10.1007/s10681-014-1103-7

Griffiths S, Wingen L, Pietragalla J, Garcia G, Hasan A, Miralles D, Calderini DF, Ankleshwaria JB, Waite ML, Simmonds J, Snape J, Reynolds M (2015) Genetic dissection of grain size and grain number trade-offs in CIMMYT wheat germplasm. PLoS ONE 10:e0118847. https://doi.org/10.1371/journal.pone.0118847

Guo Y, Sun JJ, Zhang GZ, Wang YY, Kong FM, Zhao Y, Li SS (2013) Haplotype, molecular marker and phenotype effects associated with mineral nutrient and grain size traits of TaGS1 a in wheat. Field Crops Res 154:119-125. https://doi. org/10.1016/j.fcr.2013.07.012

Hanif M, Gao FM, Liu JD, Wen WE, Zhang YJ, Rasheed A, Xia XC, He ZH, Cao SH (2016) TaTGW6-A1, an ortholog of rice TGW6, is associated with grain weight and yield in bread wheat. Mol Breed 36:1. https://doi.org/10.1007/s11032-015-0425-z

Hong YT, Chen LF, Du LP, Su ZQ, Wang JF, Ye XG, Qi L, Zhang ZY (2014) Transcript suppression of $T a G W 2$ increased grain width and weight in bread wheat. Funct Integr Genom 14:341349. https://doi.org/10.1007/s10142-014-0380-5

Hu MJ, Zhang HP, Cao JJ, Zhu XF, Wang SX, Jiang H, Wu ZY, Lu J, Chang C, Sun GL, Ma CX (2016) Characterization of an IAAglucose hydrolase gene TaTGW6 associated with grain weight in common wheat (Triticum aestivum L.). Mol Breed 36:1-11. https://doi.org/10.1007/s11032-016-0449-Z

Huang XQ, Kempf H, Ganal MW, Roder MS (2004) Advanced backcross QTL analysis in progenies derived from a cross between a German elite winter wheat variety and a synthetic wheat (Triticum aestivum L.). Theor Appl Genet 109:933-943. https://doi. org/10.1007/s00122-004-1708-7

IWGSC (2014) A chromosome-based draft sequence of the hexaploid bread wheat (Triticum aestivum) genome. Science 345:1251788. https://doi.org/10.1126/science. 1251788

Jaiswal V, Gahlaut V, Mathur S, Agarwal P, Khandelwal MK, Khurana JP, Tyagi AK, Balyan HS, Gupta PK (2015) Identification of novel SNP in promoter sequence of $T a G W 2-6 A$ associated with grain weight and other agronomic traits in wheat (Triticum aestivum
L.). PLoS ONE 10:e0129400. https://doi.org/10.1371/journal. pone. 0129400

Jia HY, Wan HS, Yang SH, Zhang ZZ, Kong ZX, Xue SL, Zhang LX, Ma ZQ (2013) Genetic dissection of yield-related traits in a recombinant inbred line population created using a key breeding parent in China's wheat breeding. Theor Appl Genet 126:21232139. https://doi.org/10.1007/s00122-013-2123-8

Jiang L, Ge M, Zhao H, Zhang TF (2015a) Analysis of heterosis and quantitative trait loci for kernel shape related traits using triple testcross population in maize. PLoS ONE 10:e0124779. https:// doi.org/10.1371/journal.pone.0124779

Jiang YM, Jiang QY, Hao CY, Hou J, Wang LF, Zhang HN, Zhang SN, Chen XH, Zhang XY (2015b) A yield-associated gene TaCWI, in wheat: its function, selection and evolution in global breeding revealed by haplotype analysis. Theor Appl Genet 128:131-143. https://doi.org/10.1007/s00122-014-2417-5

Kumar N, Kulwal PL, Balyan HS, Gupta PK (2007) QTL mapping for yield and yield contributing traits in two mapping populations of bread wheat. Mol Breed 19:163-177. https://doi.org/10.1007/ s11032-006-9056-8

Leborgne-Castel N, Jelitto-Van Dooren EPWM, Crofts AJ, Denecke J (1999) Overexpression of BiP in tobacco alleviates endoplasmic reticulum stress. Plant Cell 11:459-469. https://doi.org/10.1105/ tpc.11.3.459

Li XY, Qian Q, Fu ZM, Wang YH, Xiong GS, Zeng DL, Wang XQ, Liu XF, Teng S, Hiroshi F, Yuan M, Luo D, Han B, Li JY (2003) Control of tillering in rice. Nature 422:618-621. https://doi. org/10.1038/nature01518

Li HJ, Yang QS, Gao LL, Zhang M, Ni ZF, Zhang YR (2017) Identification of heterosis-associated stable QTLs for ear-weight-related traits in an elite maize hybrid Zhengdan 958 by design III. Front Plant Sci 8:561. https://doi.org/10.3389/fpls.2017.00561

Ling HQ, Zhao SC, Liu DC, Wang JY, Sun H, Zhang C, Fan HJ, Li D, Dong LL, Tao Y, Gao C, Wu HL, Li YW, Cui Y, Guo XS, Zheng SS, Wang B, Yu K, Liang QS, Yang WL, Lou XY, Chen J, Feng MJ, Jian JB, Zhang XF, Luo GB, Jiang Y, Liu JJ, Wang ZB, Sha YH, Zhang BR, Wu HJ, Tang DZ, Shen QH, Xue PY, Zou SH, Wang XJ, Liu X, Wang FM, Yang YP, An XL, Dong ZY, Zhang KP, Zhang XQ, Luo MC, Dvorak J, Tong YP, Wang J, Yang HM, Li ZS, Wang DW, Zhang AM, Wang J (2013) Draft genome of the wheat A-genome progenitor Triticum urartu. Nature 496:87-90. https://doi.org/10.1038/nature11997

Liu G, Jia L, Lu L, Qin D, Zhang J, Guan P, Ni Z, Yao Y, Sun Q, Peng H (2014) Mapping QTLs of yield-related traits using RIL population derived from common wheat and Tibetan semi-wild wheat. Theor Appl Genet 127(11):2415-2432. https://doi.org/10.1007/ s00122-014-2387-7

Lu P, Liang Y, Li DL, Wang ZZ, Li WB, Wang GX, Wang Y, Zhou SH, Wu QH, Xie JZ, Zhang DY, Chen YX, Li MM, Zhang Y, Sun QX, Han CG, Liu ZY (2016) Fine genetic mapping of spot blotch resistance gene $S b 3$ in wheat (Triticum aestivum). Theor Appl Genet 129:577-589. https://doi.org/10.1007/s00122-015-2649-z

Ma ZQ, Zhao DM, Zhang CQ, Zhang ZZ, Xue SL, Lin F, Kong ZX, Tian DG, Luo QY (2007) Molecular genetic analysis of five spike-related traits in wheat using RIL and immortalized $\mathrm{F}_{2}$ populations. Mol Genet Genom 277:31-42. https://doi.org/10.1007/ s00438-006-0166-0

Ma DY, Yan J, He ZH, Wu L, Xia XC (2012) Characterization of a cell wall invertase gene TaCwi-Al on common wheat chromosome 2A and development of functional markers. Mol Breed 29:43-52. https://doi.org/10.1007/s11032-010-9524-z

Ma M, Wang Q, Li ZJ, Cheng HH, Li ZJ, Liu XL, Song WN, Appels R, Zhao HX (2015) Expression of TaCYP78A3, a gene encoding cytochrome P450 CYP78A3 protein in wheat (Triticum aestivum L.), affects seed size. Plant J 83:312-325. https://doi.org/10.1111/ tpj. 12896 
Ma L, Li T, Hao CY, Wang YQ, Chen XH, Zhang XY (2016) TaGS5$3 A$, a grain size gene selected during wheat improvement for larger kernel and yield. Plant Biotechnol J 14:1269-1280. https:// doi.org/10.1111/pbi.12492

McIntosh RD, Rogers J, Morris C, Appels R, Xia X (2011) Catalogue of gene symbols for wheat: 2011 supplement. Annu Wheat Newslett 57:303-321

Mir RR, Kumar N, Jaiswal V, Girdharwal N, Prasad M, Balyan HS, Gupta PK (2012) Genetic dissection of grain weight in bread wheat through quantitative trait locus interval and association mapping. Mol Breed 29:963-972. https://doi.org/10.1007/ s11032-011-9693-4

Ni Z, Yao Y, Peng H, Hu Z, Sun Q (2013) Genomics and heterosis in hexaploid wheat. In: Chen ZJ, Birchler JA (eds) Polyploid and hybrid genomics. Wiley, Oxford. doi:https://doi. org/10.1002/9781118552872.ch6

Paul P, Awasthi A, Rai AK, Gupta SK, Prasad R, Sharma TR, Dhaliwal HS (2012) Reduced tillering in Basmati rice T-DNA insertional mutant $O S T E F 1$ associates with differential expression of stress related genes and transcription factors. Funct Integr Genom 12:291-304. https://doi.org/10.1007/s10142-012-0264-5

Pawar PM-A, Ratke C, Balasubramanian VK, Chong S-L, Gandla ML, Adriasola M, Sparrman T, Hedenström M, Szwaj K, Derba-Maceluch M, Gaertner C, Mouille G, Ezcurra I, Tenkanen M, Jönsson LJ, Mellerowicz EJ (2017) Downregulation of RWA genes in hybrid aspen affects xylan acetylation and wood saccharification. New Phytol 214(4):1491-1505. https://doi.org/10.1111/nph.14489

Ray DK, Mueller ND, West PC, Foley JA (2013) Yield trends are insufficient to double global crop production by 2050. PLoS ONE 8(6):e66428. https://doi.org/10.1371/journal.pone.0066428

Reif JC, Maurer HP, Korzun V, Ebmeyer E, Miedaner T, Wurschum T (2011) Mapping QTLs with main and epistatic effects underlying grain yield and heading time in soft winter wheat. Theor Appl Genet 123:283-292. https://doi.org/10.1007/s00122-011-1583-y

Shukla S, Singh K, Patil RV, Kadam S, Bharti S, Prasad P, Singh NK, Khanna-Chopra R (2015) Genomic regions associated with grain yield under drought stress in wheat (Triticum aestivum L.). Euphytica 203:449-467. https://doi.org/10.1007/ s10681-014-1314-y

Simmonds J, Scott P, Leverington-Waite M, Turner AS, Brinton J, Korzun V, Snape J, Uauy C (2014) Identification and independent validation of a stable yield and thousand grain weight QTL on chromosome 6A of hexaploid wheat (Triticum aestivum L.). BMC Plant Biol 14:191. https://doi.org/10.1186/s12870-014-0191-9

Simmonds J, Scott P, Brinton J, Mestre TC, Bush M, del Blanco A, Dubcovsky J, Uauy C (2016) A splice acceptor site mutation in $T a G W 2-A 1$ increases thousand grain weight in tetraploid and hexaploid wheat through wider and longer grains. Theor Appl Genet 129:1099-1112. https://doi.org/10.1007/s00122-016-2686-2

Snape JW, Foulkes MJ, Simmonds J, Leverington M, Fish LJ, Wang Y, Ciavarrella M (2007) Dissecting gene x environmental effects on wheat yields via QTL and physiological analysis. Euphytica 154:401-408. https://doi.org/10.1007/s10681-006-9208-2

Song XJ, Huang W, Shi M, Zhu MZ, Lin HX (2007) A QTL for rice grain width and weight encodes a previously unknown RINGtype E3 ubiquitin ligase. Nat Genet 39:623-630. https://doi. org/10.1038/ng2014

Su ZQ, Hao CY, Wang LF, Dong YC, Zhang XY (2011) Identification and development of a functional marker of $T a G W 2$ associated with grain weight in bread wheat (Triticum aestivum L.). Theor Appl Genet 122:211-223. https://doi.org/10.1007/s00122-010-1437-Z

Sun XY, Wu K, Zhao Y, Kong FM, Han GZ, Jiang HM, Huang XJ, Li RJ, Wang HG, Li SS (2009) QTL analysis of kernel shape and weight using recombinant inbred lines in wheat. Euphytica 165:615-624. https://doi.org/10.1007/s10681-008-9794-2

Wang RX, Hai L, Zhang XY, You GX, Yan CS, Xiao SH (2009) QTL mapping for grain filling rate and yield-related traits in RILs of the Chinese winter wheat population Heshangmai $\times$ Yu8679. Theor Appl Genet 118:313-325. https://doi.org/10.1007/ s00122-008-0901-5

Wang S, Basten C, Zeng Z (2012) Windows QTL cartographer 2.5. Department of Statistics, North Carolina State University, Raleigh. http://statgen.ncsu.edu/qtlcart/WQTLCart.htm. Accessed 16 Nov 2017

Wu XS, Chang XP, Jing RL (2012) Genetic insight into yieldassociated traits of wheat grown in multiple rain-fed environments. PLoS ONE 7:e31249. https://doi.org/10.1371/journal. pone.0031249

Xing YZ, Zhang QF (2010) Genetic and molecular bases of rice yield. Annu Rev Plant Biol 61:421-442. https://doi.org/10.1146/ annurev-arplant-042809-112209

Yang ZB, Bai ZY, Li XL, Wang P, Wu QX, Yang L, Li LQ, Li XJ (2012) SNP identification and allelic-specific PCR markers development for $T a G W 2$, a gene linked to wheat kernel weight. Theor Appl Genet 125:1057-1068. https://doi.org/10.1007/ s00122-012-1895-6

Yuan QQ, Deng ZY, Peng T, Tian JC (2012) QTL-based analysis of heterosis for number of grains per spike in wheat using DH and immortalized $\mathrm{F}_{2}$ populations. Euphytica 188:387-395. https://doi. org/10.1007/s10681-012-0694-0

Zanke CD, Ling J, Plieske J, Kollers S, Ebmeyer E, Korzun V, Argillier O, Stiewe G, Hinze M, Neumann F, Eichhorn A, Polley A, Jaenecke C, Ganal MW, Roder MS (2015) Analysis of main effect QTL for thousand grain weight in European winter wheat (Triticum aestivum L.) by genome-wide association mapping. Front. Plant Sci 6:644. https://doi.org/10.3389/fpls.2015.00644

Zhai HJ, Feng ZY, Li J, Liu XY, Xiao SH, Ni ZF, Sun QX (2016) QTL analysis of spike morphological traits and plant height in winter wheat (Triticum aestivum L.) using a high-density SNP and SSR-based linkage map. Front Plant Sci 7:1617. https://doi. org/10.3389/fpls.2016.01617

Zhang JP, Liu WH, Yang XM, Gao AN, Li XQ, Wu XY, Li LH (2011) Isolation and characterization of two putative cytokinin oxidase genes related to grain number per spike phenotype in wheat. Mol Biol Rep 38:2337-2347. https://doi.org/10.1007/ s11033-010-0367-9

Zhang XY, Chen JS, Shi CL, Chen JN, Zheng FF, Tian JC (2013) Function of $T a G W 2-6 A$ and its effect on grain weight in wheat (Triticum aestivum L.). Euphytica 192:347-357. https://doi. org/10.1007/s10681-012-0858-y

Zhang B, Liu X, Xu WN, Chang JZ, Li A, Mao XG, Zhang XY, Jing RL (2015) Novel function of a putative MOCl ortholog associated with spikelet number per spike in common wheat. Sci Rep 5:12211. https://doi.org/10.1038/srep12211

Zheng TC, Zhang XK, Yin GH, Wang LN, Han YL, Chen L et al (2011) Genetic gains in grain yield, net photosynthesis and stomatal conductance achieved in Henan Province of China between 1981 and 2008. Field Crops Res 122(3):225-233. https://doi. org/10.1016/j.fcr.2011.03.015

Zheng J, Liu H, Wang YQ, Wang LF, Chang XP, Jing RL, Hao CY, Zhang XY (2014) TEF-7A, a transcript elongation factor gene, influences yield-related traits in bread wheat (Triticum aestivum L.). J Exp Bot 65:5351-5365. https://doi.org/10.1093/jxb/eru306

Zuo JR, Li JY (2014) Molecular genetic dissection of quantitative trait loci regulating rice grain size. Annu Rev Genet 48:99-118. https:// doi.org/10.1146/annurev-genet-120213-092138 